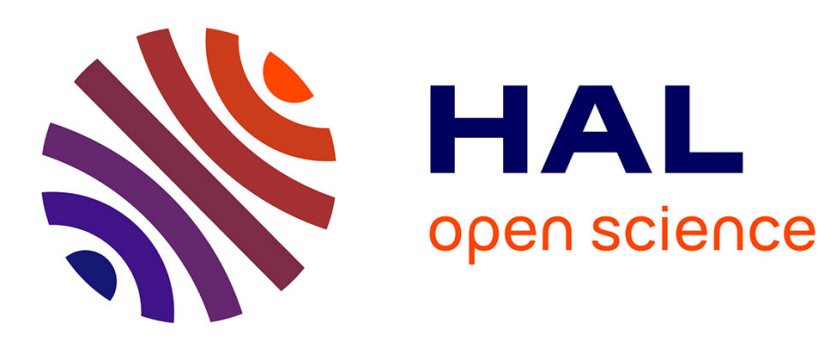

\title{
Dual norms and image decomposition models
}

\author{
Jean-François Aujol, Antonin Chambolle
}

\section{To cite this version:}

Jean-François Aujol, Antonin Chambolle. Dual norms and image decomposition models. RR-5130, INRIA. 2004. inria-00071453

\section{HAL Id: inria-00071453 \\ https://hal.inria.fr/inria-00071453}

Submitted on 23 May 2006

HAL is a multi-disciplinary open access archive for the deposit and dissemination of scientific research documents, whether they are published or not. The documents may come from teaching and research institutions in France or abroad, or from public or private research centers.
L'archive ouverte pluridisciplinaire HAL, est destinée au dépôt et à la diffusion de documents scientifiques de niveau recherche, publiés ou non, émanant des établissements d'enseignement et de recherche français ou étrangers, des laboratoires publics ou privés. 


\title{
Dual norms and image decomposition models
}

\author{
Jean-François Aujol — Antonin Chambolle
}

\section{$\mathbf{N}^{\circ} \mathbf{5 1 3 0}$}

March 2004

THÈME 3 



\title{
Dual norms and image decomposition models
}

\author{
Jean-François Aujol * ${ }^{\dagger}$, Antonin Chambolle $\S^{\ddagger}$ \\ Thème 3 - Interaction homme-machine, \\ images, données, connaissances \\ Projet Ariana
}

Rapport de recherche $\mathrm{n}^{\circ} 5130$ - March $2004-55$ pages

\begin{abstract}
Following [16], decomposition models into a geometrical component and a textured component have recently been proposed in image processing $[20,17,4,19]$. In such approaches, negative Sobolev norms have seemed to be useful to modelize oscillating patterns. In this paper, we compare the properties of various norms that are dual of Sobolev or Besov norms. We then propose a decomposition model which splits an image into three components: a first one containing the structure of the image, a second one the texture of the image, and a third one the noise. Our decomposition model relies on the use of three different semi-norms: the total variation for the geometrical componant, a negative Sobolev norm for the texture, and a negative Besov norm for the noise. We illustrate our study with numerical examples.
\end{abstract}

Key-words: Total variation minimization, $B V$, texture, noise, negative Sobolev spaces, negative Besov spaces, image decomposition

* également membre du laboratoire J.A Dieudonné, Université de Nice-Sophia-Antipolis

$\dagger$ jfaujol@sophia.inria.fr

‡MAP (UMR 7641), Ecole Polytechnique, 91128 Palaiseau Cedex, France

$\S$ antonin.chambolle@polytechnique.fr 


\section{Normes duales et modèles de décomposition d'images}

Résumé : Inspiré par [16], de nombreux modèles de décomposition d'images en une composante géométrique et une composante texturée ont été proposés en traitement d'images $[20,17,4,19]$. Dans de telles approches, les normes d'espaces de Sobolev d'exposant négatif ont paru intéressantes pour modéliser les éléments oscillants. Dans ce papier, nous comparons les propriétés de différentes normes qui sont duales de normes de Sobolev ou de Besov. Nous proposons ensuite un modèle de décomposition qui sépare une image en deux composantes, une première contenant les structures de l'image, une seconde les textures de l'image, et une troisième le bruit. Notre modèle de décomposition repose sur l'utilisation de trois semi-normes différentes: la variation totale pour la composante géométrique, une norme de Sobolev négative pour la texture, et une norme de Besov négative pour le bruit. Nous illustrons notre étude par des exemples numériques.

Mots-clés : Minimisation de la variation totale, $B V$, texture, bruit, espace de Sobolev d'exposant négatif, espace de Besov d'exposant négatif, décomposition d'images 


\section{Contents}

1 Motivation $\mathbf{5}$

1.1 Decomposition models ............... 5

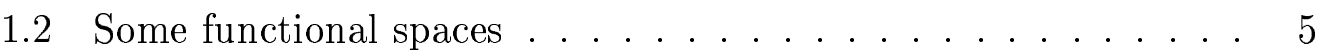

1.3 Overview of the paper ............ 8

2 Discretization $\quad 8$

2.1 Notations .................... 8

2.1.1 Total variation ................. 9

2.1.2 Sobolev semi-norms . . . . . . . . . . . . 9

2.1.3 G-norm .................. 10

2.2 Total variation minimization as a projection . . . . . . . 11

3 Computing dual norms $\quad 12$

3.1 Computing $G$ norm . . . . . . . . . . . . . . . . 12

3.2 Application to a decomposition algorithm . . . . . . . . 13

3.2.1 Introduction . . . . . . . . . . . . . . 13

3.2.2 Choice of the $\mu$ parameter . . . . . . . . . . 14

3.3 Computing $-1, p$ norms $(p \in(1,+\infty)) \ldots \ldots \ldots$

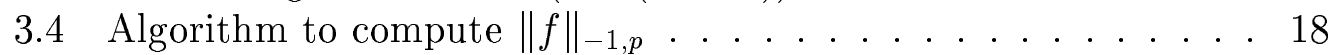

3.5 Numerical results . . . . . . . . . . . . . . . . 18

$3.6-1,2$ norm of a white gaussian noise . . . . . . . . . . 21

3.7 An algorithm to solve the Osher-Solé-Vese model . . . . . . . 27

4 Minimization of dual norms $\quad 28$

4.1 Some convex analysis . . . . . . . . . . . . . 28

4.2 Application to $u+v$ models . . . . . . . . . . . . 32

5 A new $u+v$ algorithm 33

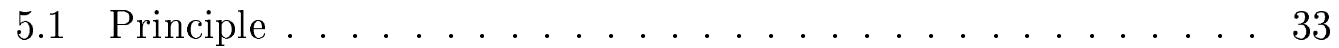

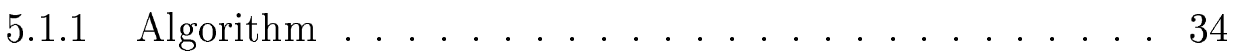

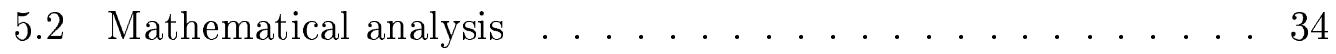

5.3 Numerical results and comments . . . . . . . . . . 35

$\mathrm{RR} \mathrm{n}^{\circ} 5130$ 
$6 u+v+w$ model $\quad 40$

6.1 Presentation . . . . . . . . . . . . . . 40

6.2 Algorithm . . . . . . . . . . . . . . . . . 43

6.3 Limit problem . . . . . . . . . . . . . . . . . . . . . 47

6.4 Numerical results . . . . . . . . . . . . . . . . . . . 47 


\section{Motivation}

\subsection{Decomposition models}

Decomposing an image into meaningful components is one of the major goals of image processing. A first range of models are denoising models: in such models, the image is assumed to have been corrupted by a noise, and what we want is to remove the noise. There are mainly two kinds of approaches. A first one is based on a process on the wavelet coefficients of the image $[9,14,8,13$, $12,16]$. A second one is based on the resolution of the PDE associated to the minimization of an energy involving some norm of the gradient $[18,7,16]$.

More recently, following [16], decomposition models into a geometrical component and a textured component have been proposed [20, 17, 4, 19]. The first three models rely on the use of a negative Sobolev norm to characterize textures. [19] uses appropriate dictionaries to characterize textures, and at the end even gets a decomposition of the image into three components: a first one containing the geometrical information of the image, a second one the texture, and a last one the noise. In this paper, we would like to study more deeply the characteristic of negative Sobolev norms, and then propose a decomposition model which would split an image into three components: a first one containing the structure of the image, a second one the texture of the image, and a third one the noise.

\subsection{Some functional spaces}

Although we will work only in the discrete setting in the rest of the paper, we first introduce various functional spaces that have been considered in image processing for describing piecewise smooth or oscillatory components in images. Throughout this subsection, we denote by $\Omega$ an open connected set of $\mathbb{R}^{2}$ with Lipschitz boundary.

The use of different functional spaces is a common tool in image processing, especially in minimization processes (see [3, 16] and references). For instance, the space $B V$ of functions with bounded variation is often used. It enjoys the

$\mathrm{RR} \mathrm{n}^{\circ} 5130$ 
nice feature of containing functions with discontinuities along lines, that can represent the edges in an image. We recall here its definition [2].

Definition 1.1. $B V(\Omega)$ is the subspace of functions $u \in L^{1}(\Omega)$ such that the following quantity, called the Total Variation of $u$, is finite:

$$
J(u)=\sup \left\{\int_{\Omega} u(x) \operatorname{div}(\xi(x)) d x / \xi \in C_{c}^{1}\left(\Omega ; \mathbb{R}^{2}\right),\|\xi\|_{L^{\infty}(\Omega)} \leq 1\right\}
$$

The total variation plays a key-role in the Rudin-Osher-Fatemi (ROF) model [18]. Yves Meyer has recently investigated this model in [16]. Unsatisfied with the results, he has introduced a space $G$ for oscillating patterns. This space happens to be very close to the dual space of $B V$. In such a space, oscillating patterns have a small norm: this a usefull property to catch them with an energy minimization process. We recall here the definition of $G$ :

Definition 1.2. $G$ is the Banach space composed of the distributions $f$ which can be written

$$
f=\partial_{1} g_{1}+\partial_{2} g_{2}=\operatorname{div}(g)
$$

with $g_{1}$ and $g_{2}$ in $L^{\infty}(\Omega)$. On $G$, the following norm is defined:

$$
\|f\|_{G}=\inf \left\{\|g\|_{L^{\infty}\left(\Omega, \mathbb{R}^{2}\right)} / f=\operatorname{div}(g)\right\}
$$

with $\|g\|_{L^{\infty}\left(\Omega, \mathbb{R}^{2}\right)}=\operatorname{ess}_{\sup _{x \in \Omega}} \sqrt{\left|g_{1}\right|^{2}+\left|g_{2}\right|^{2}}(x)$

In fact, with these notations, $G$ is exactly $W^{-1, \infty}(\Omega)$, the dual space of $W_{0}^{1,1}(\Omega)[1]$. And it is shown in the discrete case in [4] that $\|\cdot\|_{G}$ is the polar semi-norm of $J($.$) (see Proposition 2.1).$

In [16], the author has proposed a new decomposition algorithm: it splits an original image $f$ into a sum $u+v$ of a bounded variation component and a component containing the oscillating part of the image by minimizing:

$$
J(u)+\|v\|_{G}
$$

But, due to the $G$ norm, this decomposition is quite difficult to compute. Algorithms have been proposed to solve Meyer's problem or close ones [20,4,5]. These works have confirmed that $G$ is a very good space to modelize oscillating patterns, and especially textures. 
In [17], the $G$ norm is replaced by another negative Sobolev norm. For the reader convenience, we recall here the definition of a Sobolev space(see [1]):

Definition 1.3. If $p \in(1,+\infty)$, then $W^{1, p}(\Omega)$ is the space of functions in $L^{p}(\Omega)$, and such that their distributional derivative also belongs to $L^{p}(\Omega)$. $W_{0}^{1, p}(\Omega)$ is the closure of $C_{c}^{1}(\Omega)$ in $W^{1, p}(\Omega)$. Let $q=\frac{p}{p-1}\left(\right.$ so that $\left.\frac{1}{p}+\frac{1}{q}=1\right)$. We denote by $W^{-1, q}(\Omega)$ the dual space of $W_{0}^{1, p}(\Omega)$.

Oscillating patterns have a small norm in a negative Sobolev space. And such a norm may be easier to minimize than the $G$ norm.

In [16], the author also suggests the use of another dual space to model oscillating patterns: $E=\dot{B}_{-1, \infty}^{\infty}$. This is the dual space of $\dot{B}_{1,1}^{1}$.

Definition 1.4. $\dot{B}_{1,1}^{1}$ is the usual homogeneous Besov space (see [16]). Let $\psi_{j, k}$ an orthonormal base composed of smooth and compactly supported wavelets. $\dot{B}_{1,1}^{1}$ is a subspace of $L^{2}\left(\mathbb{R}^{2}\right)$, and a function $f$ belongs to $\dot{B}_{1,1}^{1}$ if and only if:

$$
\sum_{j \in \mathbb{Z}} \sum_{k \in \mathbb{Z}^{2}}\left|c_{j, k}\right| 2^{j / 2}<+\infty
$$

where $c_{j, k}$ are the wavelet coefficients of $f$.

Definition 1.5. The dual space of $\dot{B}_{1,1}^{1}$ is the Banach space $E=\dot{B}_{-1, \infty}^{\infty}$. It is characterized by the fact that the wavelet coefficients of a generalized function in $E=\dot{B}_{-1, \infty}^{\infty}$ belong to $l^{\infty}\left(\mathbb{Z} \times \mathbb{Z}^{2}\right)$.

In practice, we will use the Haar wavelet (resp. the Daubechie wavelet with eight vanishing moments) denoted by Haar (resp. Daub8) [15].

Remark: We have the following result (stated in [16]):

$$
\dot{B}_{1,1}^{1} \subset \dot{B V} \subset L^{2} \subset G \subset E=\dot{B}_{-1, \infty}^{\infty}
$$

where $\dot{B V}$ is the homogeneous version of $B V: \dot{B V}=B V /\{u \in B V / \nabla u=0\}$. In fact, we will see that $B V$ is well adapted for the geometrical part of an image, $G$ for the texture part and $E=\dot{B}_{-1, \infty}^{\infty}$ for the noise.

$\mathrm{RR} \mathrm{n}^{\circ} 5130$ 
In the rest of the paper, we will then introduce the discrete versions of the norms of these spaces

\subsection{Overview of the paper}

The aim of this work is to construct a model which decompose an image into three components, a first one containing the geometrical information, a second one containing the texture, and a last one containing the noise. To separate these components, we want to try to find appropriate norms that are well adapted to measure the relative importance of each components. As suggested in [16], we are going to use dual norms of the standard Sobolev norms to characterize the oscillating patterns.

In the first part of this paper, we introduce the discrete framework in which we will place ourself, and which is an appropriate one in image processing. In a second part, we compute dual norms used in [16, 20, 17, 4]. This will tells us which dual norms are appropriate to capture oscillating patterns. Then, in a third part, we introduce a mathematical framework to minimize dual norms. This will precise the study of the second part. In the fourth part, we propose a new $u+v$ model, which happens to be a powerful denoising model. And in the last part, we finally construct our $u+v+w$ model and show some numerical experiments.

\section{Discretization}

\subsection{Notations}

From now on, we consider the discrete case We take here the same notations as in [6]. The image is a two dimension vector of size $N \times N$. We denote by $X$ the Euclidean space $\mathbb{R}^{N \times N}$, and $Y=X \times X$. The space $X$ will be endowed with the scalar product $(u, v)_{X}=\sum_{1 \leq i, j \leq N} u_{i, j} v_{i, j}$ and the norm $\|u\|_{X}=\sqrt{(u, u)_{X}}$. We will also use the following norms $(p \geq 1)$ :

$$
\|u\|_{p}=\left(\sum_{1 \leq i, j \leq N}\left|u_{i, j}\right|^{p}\right)^{1 / p}
$$

(remark that $\left.\|u\|_{X}=\|u\|_{2}\right)$. 


\subsubsection{Total variation}

To define a discrete total variation, we introduce a discrete version of the gradient operator. If $u \in X$, the gradient $\nabla u$ is a vector in $Y$ given by: $(\nabla u)_{i, j}=\left((\nabla u)_{i, j}^{1},(\nabla u)_{i, j}^{2}\right)$. with

$$
(\nabla u)_{i, j}^{1}= \begin{cases}u_{i+1, j}-u_{i, j} & \text { if } i<N \\ 0 & \text { if } i=N\end{cases}
$$

and

$$
(\nabla u)_{i, j}^{2}= \begin{cases}u_{i, j+1}-u_{i, j} & \text { if } j<N \\ 0 & \text { if } j=N\end{cases}
$$

The discrete total variation of $u$ is then defined by:

$$
J(u)=\sum_{1 \leq i, j \leq N}\left|(\nabla u)_{i, j}\right|
$$

\subsubsection{Sobolev semi-norms}

We also use the following norms $(p \geq 1)$ :

$$
\|\nabla u\|_{p}=\left(\sum_{1 \leq i, j \leq N}\left|\nabla u_{i, j}\right|^{p}\right)^{1 / p}
$$

(remark that $\|\nabla u\|_{Y}=\|\nabla u\|_{2}$ and $\left.J(u)=\|\nabla u\|_{1}\right)$. We can now introduce the following semi-norm:

$$
\|u\|_{1, p}=\|\nabla u\|_{p}
$$

This is a norm on the space $X_{0}=\left\{u \in X, \sum_{i, j} u_{i, j}=0\right\}$. It can be seen as a discrete version of a Sobolev norm.

We consider the polar semi-norm associated to $(2.6)$ (with $1 / p+1 / q=1$ ):

$$
\|v\|_{-1, p}=\sup _{\|u\|_{1, q} \leq 1}(v, u)_{X}=\sup _{\|u\|_{1, q}=1}(v, u)_{X}=\sup _{\|\nabla u\|_{q}=1}(v, u)_{X}
$$

This is the discrete norm we use for negative Sobolev spaces.

RR $n^{\circ} 5130$ 


\subsubsection{G-norm}

We also introduce a discrete version of the divergence operator. We define it by analogy with the continuous setting by $\operatorname{div}=-\nabla^{*}$ where $\nabla^{*}$ is the adjoint of $\nabla$ : that is, for every $p \in Y$ and $u \in X,(-\operatorname{div} p, u)_{X}=(p, \nabla u)_{Y}$. It is easy to check that:

$$
(\operatorname{div}(p))_{i, j}=\left\{\begin{array}{ll}
p_{i, j}^{1}-p_{i-1, j}^{1} & \text { if } 1<i<N \\
p_{i, j}^{1} & \text { if } \mathrm{i}=1 \\
-p_{i-1, j}^{1} & \text { if } \mathrm{i}=\mathrm{N}
\end{array}+ \begin{cases}p_{i, j}^{2}-p_{i, j-1}^{2} & \text { if } 1<j<N \\
p_{i, j}^{2} & \text { if } \mathrm{j}=1 \\
-p_{i, j-1}^{2} & \text { if } \mathrm{j}=\mathrm{N}\end{cases}\right.
$$

From now on, we will use these discrete operators.

We are now in position to introduce the discrete version of Meyer's space $G$.

\section{Definition 2.1.}

$$
G=\{v \in X / \exists g \in Y \text { such that } v=\operatorname{div}(g)\}
$$

and if $v \in G$ :

$$
\begin{aligned}
& \|v\|_{G}=\inf \left\{\|g\|_{\infty} / v=\operatorname{div}(g),\right. \\
& \left.\quad g=\left(g^{1}, g^{2}\right) \in Y,\left|g_{i, j}\right|=\sqrt{\left(g_{i, j}^{1}\right)^{2}+\left(g_{i, j}^{2}\right)^{2}}\right\}
\end{aligned}
$$

where $\|g\|_{\infty}=\max _{i, j}\left|g_{i, j}\right|$.

Moreover, we will denote:

$$
B_{G(\mu)}=\mu B_{G}=\left\{v \in G /\|v\|_{G} \leq \mu\right\}
$$

We recall that the Legendre-Fenchel transform of $F$ is given by $F^{*}(v)=$ $\sup _{u}(u, v)_{X}-F(u)$ (see [10]). The two following results are proved in [4].

\section{Proposition 2.1.}

$$
J(u)=\sup _{v \in B_{G}}(u, v)_{X}
$$

and

$$
\|v\|_{G}=\sup _{J(u) \leq 1}(u, v)_{X}
$$


We see that $J($.$\left.) (resp. \|.\|_{G}\right)$ is the polar of $\|\cdot\|_{G}(\operatorname{resp} . J()$.$) .$

Proposition 2.2. The space $G$ identifies with the following subspace:

$$
X_{0}=\left\{v \in X / \sum_{i, j} v_{i, j}=0\right\}
$$

\subsection{Total variation minimization as a projection}

Introduction: Since $J$ defined by (1.1) is homogeneous of degree one (i.e. $J(\lambda u)=\lambda J(u) \forall u$ and $\lambda>0$ ), it is then standard (see [10]) that $J^{*}$ is the indicator function of some closed convex set, which turns out to be the set $B_{G}$ defined by $(2.11)$ :

$$
J^{*}(v)=\chi_{B_{G}}(v)= \begin{cases}0 & \text { if } v \in B_{G} \\ +\infty & \text { otherwise }\end{cases}
$$

This can be checked out easily (see [6] for details). In [6], the author proposes a nonlinear projection algorithm to minimize the ROF model. The problem is:

$$
\inf _{u \in X}\left(J(u)+\frac{1}{2 \lambda}\|f-u\|_{X}^{2}\right)
$$

We have the following result, which comes from standard convex duality theory [10]:

Proposition 2.3. ([6]): The solution of (2.16) is given by:

$$
u=f-P_{\lambda B_{G}}(f)
$$

where $P$ is the orthogonal projector on $\lambda B_{G}$ (defined by (2.11)).

We use the following algorithm to compute $P_{\lambda B_{G}}(f)$. It indeed amounts to finding:

$$
\min \left\{\|\lambda \operatorname{div}(p)-f\|_{X}^{2}: p /\left|p_{i, j}\right| \leq 1 \forall i, j=1, \ldots, N\right\}
$$

This problem can be solved by a fixed point method:

$$
p^{0}=0
$$

$\mathrm{RR} \mathrm{n}^{\circ} 5130$ 
and

$$
p_{i, j}^{n+1}=\frac{p_{i, j}^{n}+\tau\left(\nabla\left(\operatorname{div}\left(p^{n}\right)-f / \lambda\right)\right)_{i, j}}{1+\tau\left|\left(\nabla\left(\operatorname{div}\left(p^{n}\right)-f / \lambda\right)\right)_{i, j}\right|}
$$

In [6] is given a sufficient condition ensuring the convergence of the algorithm: it is shown that as long as $\tau \leq 1 / 8$, then $\lambda \operatorname{div}\left(p^{n}\right)$ converges to $P_{\lambda B_{G}}(f)$ as $n \rightarrow+\infty$.

\section{Computing dual norms}

Contrarily to the computation of the $E$ norm which is easily obtained once the wavelet decomposition of the image has been performed, the other dual norms are less easy to compute.

\subsection{Computing $G$ norm}

We use the projection algorithm (2.20).

Let $f$ be an image with zero mean. We remark that if $\lambda \geq 0$, then:

$$
f=P_{\lambda B_{G}}(f)
$$

if and only if $\|f\|_{G} \leq \lambda$. Thus one just need to compute the norm of $f-P_{\lambda B_{G}}(f)$ to know if $\lambda$ is larger (if the norm is zero) or smaller (if the norm is positive) than $\|f\|_{G}$. Thus we can find $\|f\|_{G}$ by dichotomy.

\section{Algorithm:}

1. Initialization: $a_{0}=0$ and $b_{0}=M$, where $M$ is assumed to be such that $\|f\|_{G} \leq M$.

2. Iterations: Set $c_{n+1}=\frac{a_{n}+b_{n}}{2}$. Compute $P_{c_{n+1} B_{G}}(f)$ by using (2.20).

If $P_{c_{n+1} B_{G}}(f) \neq f$, this means that $c_{n+1} \leq\|f\|_{G}$. Thus set $a_{n+1}=c_{n+1}$ and $b_{n+1}=b_{n}$.

Otherwise (i.e. if $P_{c_{n+1} B_{G}}(f)=f$ ), this means that $c_{n+1}>\|f\|_{G}$. Thus set $b_{n+1}=c_{n+1}$ and $a_{n+1}=a_{n}$.

3. Stopping test: we stop if $\left|b_{n}-a_{n}\right| \leq \epsilon$. 
Nevertheless, in our numerical tests, we have found that this method does not give a precise approximation of the $G$-norm of an image.

\subsection{Application to a decomposition algorithm}

\subsubsection{Introduction}

In [16], Meyer has introduced a new model to split a given image $f$ into a sum $u+v$ of a bounded variation component and a component containing the oscillating part of the image. This model has been first successfully implemented by Vese and Osher [20]. A different approach has been proposed in [4, 5]. We will focus on this one, where the decomposition is computed by minimizing a convex functional which depends on the two variables $u$ and $v$, alternatively in each variable. Each minimization is based on the projection algorithm (2.20).

The problem to solve is the following ( $f$ being the degraded image):

$$
\inf _{(u, v) \in X \times \mu B_{G}}\left(J(u)+\frac{1}{2 \lambda}\|f-u-v\|_{X}^{2}\right)
$$

where

$$
\mu B_{G}=\left\{v \in G /\|v\|_{G} \leq \mu\right\}
$$

and $\|v\|_{G}$ is defined by (1.3), and $J(u)$ by (1.1).

The algorithm to solve this problem used in $[4,5]$ needs thus the two parameters $\lambda$ and $\mu$. The parameter $\lambda$ controls the $L^{2}$-norm of the residual $f-u-v$. The smaller $\lambda$ is, the smaller the $L^{2}$ norm of the residual $f-u-v$ is. The larger $\mu$ is, the more $v$ contains information, and therefore the more $u$ is averaged. In fact, the choice of $\lambda$ is easy. One just needs to set it very small. For instance, in all the examples presented hereafter, we have chosen $\lambda=0.1$, and found out a maximum norm for $f-u-v$ of about 0.5 (for values ranging from 0 to 255). But the $\mu$ parameter is much harder to tune. It controls the $G$ norm of the oscillating component $v$. Some examples of the value of such a norm are displayed in Subsection 3.5.

$\mathrm{RR} \mathrm{n}^{\circ} 5130$ 


\subsubsection{Choice of the $\mu$ parameter}

Assume the image $u$ has been degraded by a gaussian noise of variance $\sigma^{2}$. We would like to remove that noise: hence we want the $v$ component to be a white noise of variance $\sigma^{2}$.

In the ROF method [18], it is suggested to minimize the total variation with the constraint that the residual has exactly a variance of $\sigma^{2}$. If we want to do the same for the algorithm of [4], we have to know the $G$ norm of a Gaussian norm of known standard deviation. Thanks to the homogeneity of the norm, $\|n\|_{G} / \sigma$ should be roughly constant (at least when the number of points is large enough, given the size of the image), however, the strong nonlinearity of the $G$ norm does not allow us to check rigorously this fact (in other words, that this "constant" is quite insensitive to the particular realization of the Gaussian noise we consider).

We have computed the $G$ norm of white gaussian noise images (of variance $\sigma^{2}$ ) with the algorithm explained in subsection 3.1. For instance, we have checked that the $G$ norm of a white gaussian noise with variance $\sigma=1$ is very close to 1.6 for an image of size $128^{*} 128$.

Knowing this, we can then compute the $G$ norm of any white gaussian noise $n$ of size $128^{*} 128$, provided we kow its variance $\sigma^{2}$ :

$$
\|n\|_{G} \sim 1.6 \sigma
$$

This gives the value of the $\mu$ parameter for the algorithm of [4]. Figure 1 shows how this choice of the parameter $\mu$ performs on a particular case.

\subsection{Computing $-1, p$ norms $(p \in(1,+\infty))$}

Let $p \in(1,+\infty)$. Let $f$ be a function with zero mean (i.e. $\left.\sum_{1 \leq i, j \leq N} f_{i, j}=0\right)$.

We want to compute $\|f\|_{-1, p}$ (see (2.7)). Let us set $q=\frac{p}{p-1}$ (so that $\left.\frac{1}{p}+\frac{1}{q}=1\right)$.

Let us recall that $\|u\|_{1, q}=\|\nabla u\|_{q}$ (see (2.6)) and:

$$
\|f\|_{-1, p}=\sup _{\|u\|_{1, q} \leq 1}(f, u)_{X}=\sup _{\|u\|_{1, q}=1}(f, u)_{X}=\sup _{\|\nabla u\|_{q}=1}(f, u)_{X}
$$

We look at the following minimization problem:

$$
\min _{u \in X}\left(F(u)-(f, u)_{X}\right)
$$



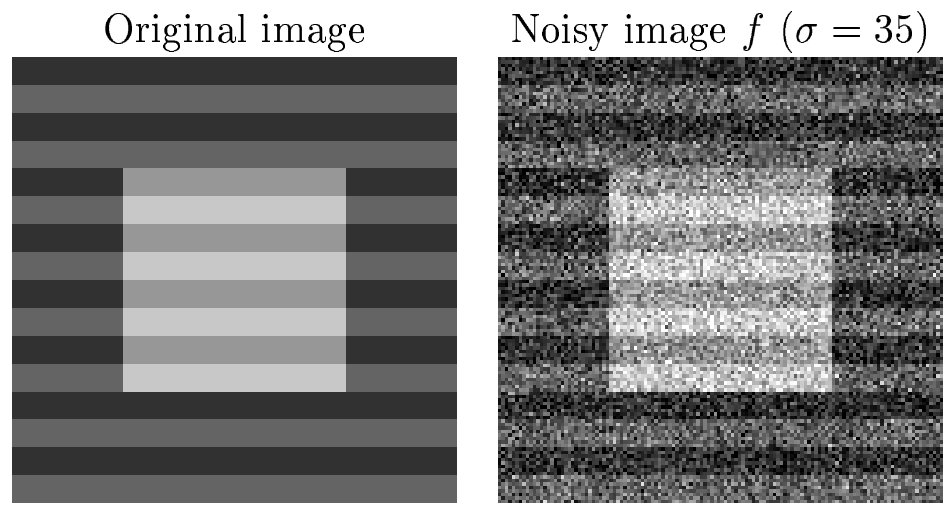

Restored image $(u[4])$

Noise $(v[4])$

$$
\lambda=0.1
$$

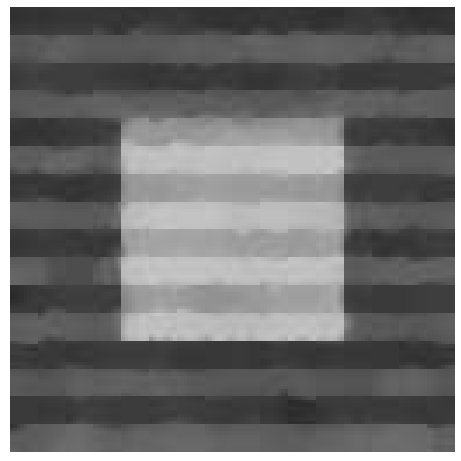

$\mu=1.6 * \sigma=56$

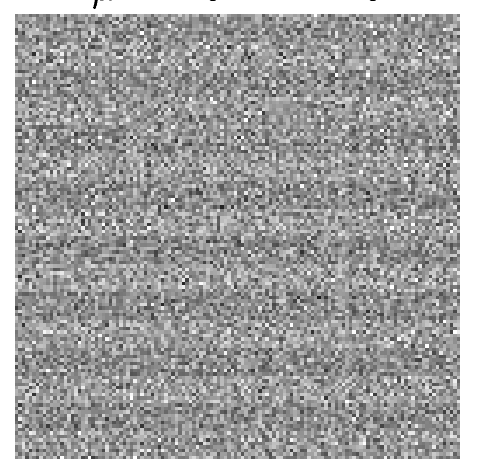

Restored image $\left(u_{R O F}\right)$ $(\sigma=35)$

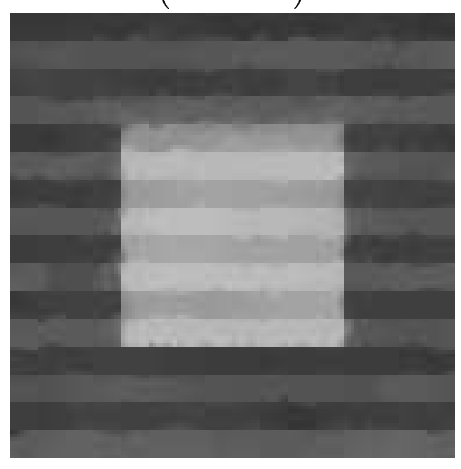

Noise $\left(v_{R O F}\right)$

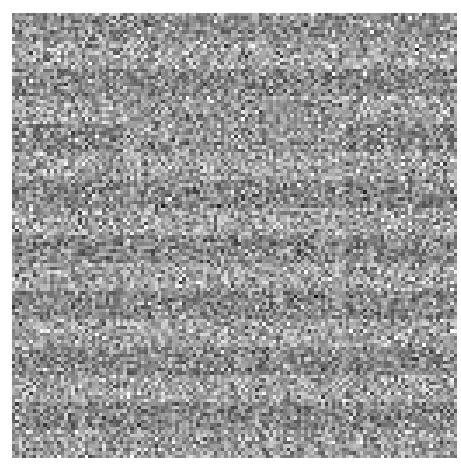

Figure 1: Denoising

$\mathrm{RR} \mathrm{n}^{\circ} 5130$ 
where

$$
F(u)=\frac{1}{q} \sum_{1 \leq i, j \leq N}\left|\nabla u_{i, j}\right|^{q}=\frac{1}{q}\|\nabla u\|_{q}^{q}
$$

By analogy with the continuous setting, we define a discrete Laplacian operator by setting $\Delta u=\operatorname{div}(\nabla u)$.

Proposition 3.1. If $\tilde{u}$ is the minimizer of (3.6), then we have for $1 \leq i, j \leq$ $N$ :

$$
\left(\operatorname{div}\left(\left|\nabla \tilde{u}_{i, j}\right|^{q-2} \nabla \tilde{u}_{i, j}\right)\right)_{i, j}=f_{i, j}
$$

Moreover, we also have:

$$
\|f\|_{-1, p}^{p}=\|\nabla \tilde{u}\|_{q}^{q}=\sum_{1 \leq i, j \leq N}\left|\nabla \tilde{u}_{i, j}\right|^{q}=q F(\tilde{u})
$$

In particular, when $p=2$, we get:

$$
\Delta \tilde{u}=f
$$

and

$$
\|f\|_{-1,2}=\|\nabla \tilde{u}\|_{Y}=\sqrt{\sum_{1 \leq i, j \leq N}\left|\nabla \tilde{u}_{i, j}\right|^{2}}
$$

Proof: (3.8) is the Euler-Lagrange equation of (3.6), and its computation is standard once one has remarked that $\left(F^{\prime}(u), h\right)_{X}=|\nabla u|^{q-2}(\nabla u, \nabla h)_{X}$. 
We concentrate now on (3.9). It is well known that the Legendre-Fenchel transform of $\frac{1}{q}\|f\|_{1, q}^{q}$ is $\frac{1}{p}\|f\|_{-1, p}^{p}$, as shown by the following computation:

$$
\begin{aligned}
\left(\frac{1}{q}\|f\|_{1, q}^{q}\right)^{*} & =\sup _{u}\left((f, u)_{X}-\frac{1}{q}\|\nabla u\|_{q}^{q}\right) \\
& =\sup _{\left\{t \geq 0, u /\|\nabla u\|_{q}=1\right\}}\left((f, t u)_{X}-\frac{t^{q}}{q}\|\nabla u\|_{q}^{q}\right) \\
& =\sup _{\left\{t \geq 0, u /\|\nabla u\|_{q}=1\right\}}\left(t(f, u)_{X}-\frac{t^{q}}{q}\right) \\
& =\sup _{t \geq 0}\left(\sup _{\|\nabla u\|_{q}=1} t(f, u)_{X}-\frac{t^{q}}{q}\right) \\
& =\sup _{t \geq 0}\left(t\|f\|_{-1, p}-\frac{t^{q}}{q}\right) \\
& =\frac{1}{p}\|f\|_{-1, p}^{p}
\end{aligned}
$$

We therefore deduce that

$$
-\frac{1}{p}\|f\|_{-1, p}^{p}=\min _{u}\left(F(u)-(f, u)_{X}\right)=F(\tilde{u})-(f, \tilde{u})_{X}
$$

The solution $\tilde{u}$ of (3.6) satisfies

$$
F(\tilde{u})=\frac{1}{q} \sum_{1 \leq i, j \leq N}\left|\nabla \tilde{u}_{i, j}\right|^{q}=\frac{1}{q}\|\nabla \tilde{u}\|_{q}^{q}
$$

and by Euler's identity we have $\left(F^{\prime}(\tilde{u}), \tilde{u}\right)_{X}=q F(\tilde{u})$. We thus deduce that:

$$
(f, \tilde{u})_{X}=q F(\tilde{u})
$$

From (3.12), we get:

$$
-\frac{1}{p}\|f\|_{-1, p}^{p}=(1-q) F(\tilde{u})=-\frac{q}{p} F(\tilde{u})
$$

$\mathrm{RR} \mathrm{n}^{\circ} 5130$ 
Table 1: A striking example

\begin{tabular}{|c|c|c|c|c|c|}
\hline Images & $T V$ & $L^{2}$ & $-1,2$ & $G$ & $E$ (Daub10, order 4) \\
\hline \hline textured image & 1000000 & 9500 & 33000 & 360 & 749 \\
\hline geometric image & 64600 & 9500 & 300000 & 2000 & 355 \\
\hline Gaussian noise $(\sigma=85)$ & 2100000 & 9500 & 9100 & 120 & 287 \\
\hline
\end{tabular}

\subsection{Algorithm to compute $\|f\|_{-1, p}$}

From Propositions 3.1 , we see that a way to compute $\|f\|_{-1, p}$ is by minimizing (3.6) and by using the formula:

$$
\|f\|_{-1, p}^{p}=\frac{1}{q}\|\nabla \tilde{u}\|_{q}^{q}=\frac{1}{q} \sum_{1 \leq i, j \leq N}\left|\nabla u_{i, j}\right|^{q}=q F(\tilde{u})
$$

We can minimize the convex function $F$ by steepest descent.

Remark: when $p=2$ then it is immediate to compute $\|f\|_{-1,2}=\sqrt{\left(-f, \Delta^{-1} f\right)_{X}}$ by using the discrete Fourier transform (see the end of subsection 3.6).

\subsection{Numerical results}

\section{A striking example:}

Table 1 clearly illustrates the superiority of the $G$ norm over the $L^{2}$ norm to capture oscillating patterns in minimization processes, as claimed in [16]. It also illustrates why the use of the $E$ norm is well adapted to separate the noise.

\section{Other computations:}

Regarding the norms of the images of white gaussian noises, we have checked that our results do not depend on the way our Gaussian noise is simulated: for each white gaussian noise, we have created ten different images 

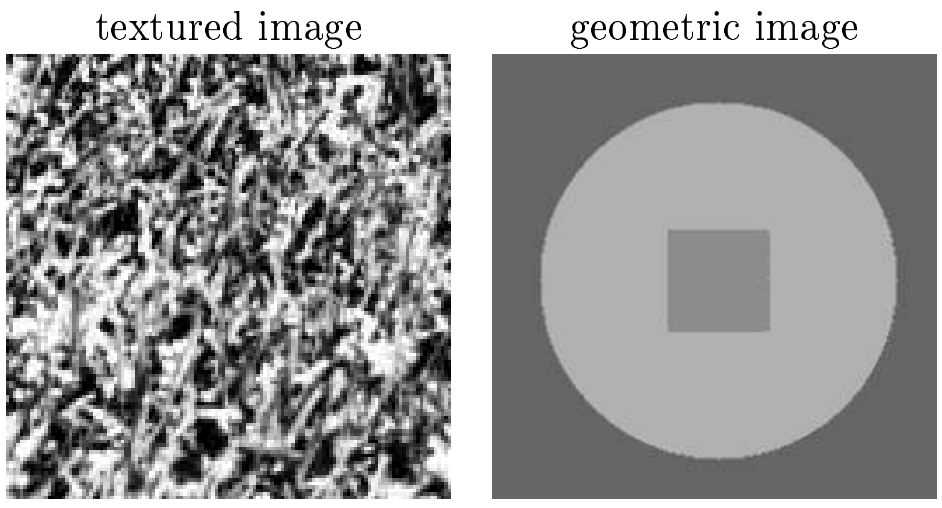

Gaussian noise $(\sigma=85)$

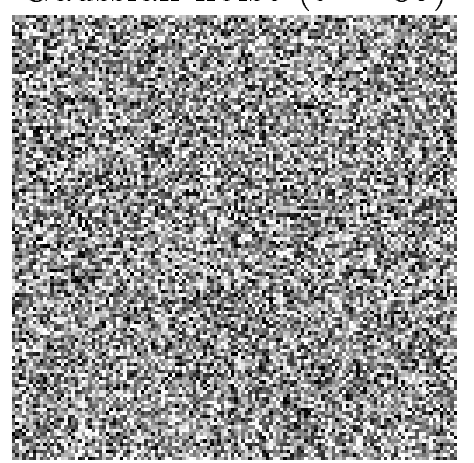

Figure 2: A striking example

Table 2: Images of size $128^{*} 128$

\begin{tabular}{|c|c|c|c|c|c|}
\hline Images & $T V$ & $L^{2}$ & $G$ & $-1,2$ & $E$ (Daub10, order3) \\
\hline \hline param1 & 1000000 & 9500 & 360 & 33000 & 730 \\
\hline param2 & 800000 & 9500 & 300 & 26000 & 690 \\
\hline param3 & 980000 & 9450 & 320 & 30000 & 890 \\
\hline param4 & 1100000 & 9450 & 290 & 22000 & 630 \\
\hline circles & 18000 & 3000 & 2000 & 18000 & 280 \\
\hline G20 & 570000 & 2500 & 31 & 2300 & 73 \\
\hline G30 & 850000 & 3900 & 50.5 & 3700 & 110 \\
\hline G50 & 1400000 & 6300 & 84 & 6100 & 180 \\
\hline G85 & 2100000 & 9500 & 120 & 9100 & 290 \\
\hline
\end{tabular}

$\mathrm{RR} \mathrm{n}^{\circ} 5130$ 
param1

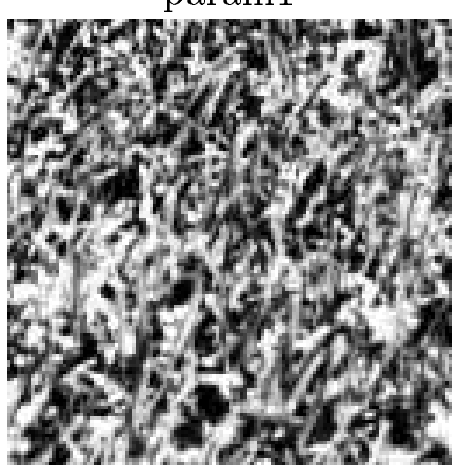

param 3

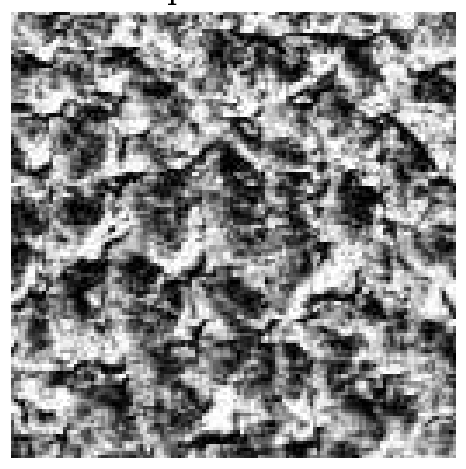

param 2

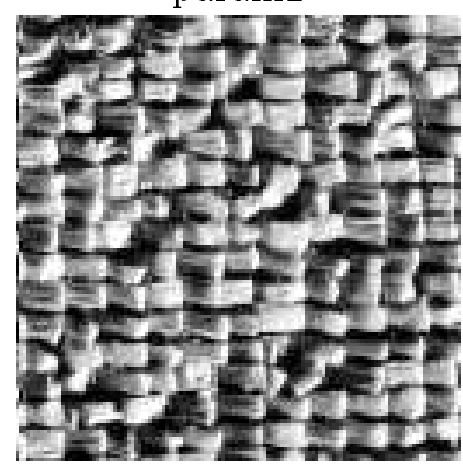

param 4

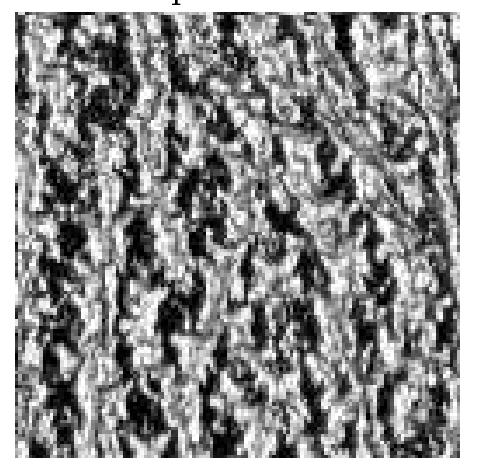

Figure 3: Textured images

circles

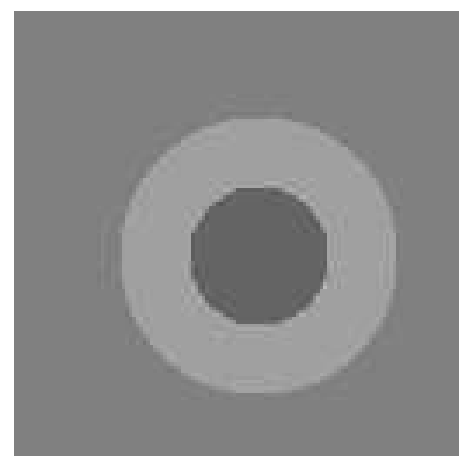

Figure 4: BV image 

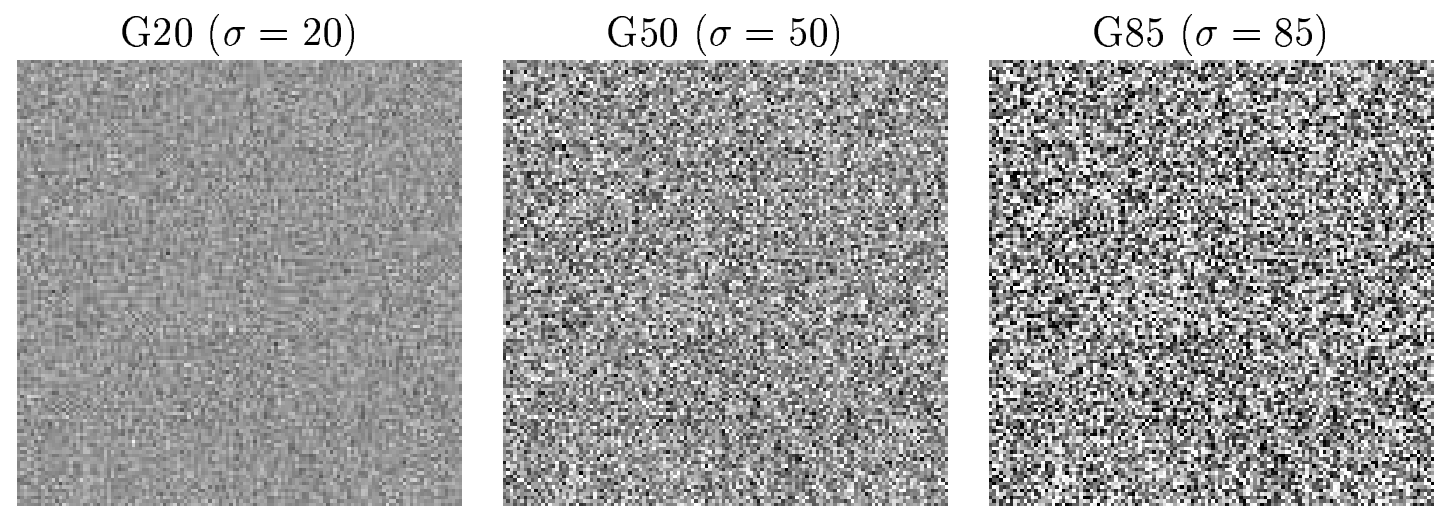

Figure 5: Gaussian noise images

on which we computed the norms. We have noticed that the variances of the $G$ and $-1,2$ norms are slightly larger than the ones of the $T V$ and $L^{2}$ norms, but that they nevertheless remain quite small with respect to their mean values (see also Table 4 at the end of subsection 3.6).

As can be seen on Table 2, there appears a very strong correlation between the $L^{2}$ norm and the $-1,2$ norm of the image of a white gaussian noise. This will be confirmed by the results of the next subsection.

\section{$3.6-1,2$ norm of a white gaussian noise}

We consider an image $n=\left(n_{i, j}\right)$ of size $N \times N$. To simplify, we use periodic conditions in this subsection (but only in this subsection). We therefore not use (2.2), (2.3) and (2.8), but a discrete gradient and a discrete divergence with periodic conditions. We assume that $n$ is an image of a white gaussian noise; i.e. for all $(i, j), n_{i, j}$ follows a gaussian law whose probability density function reads:

$$
f(x)=\frac{1}{\sqrt{2 \pi \sigma^{2}}} \exp \left(\frac{-x^{2}}{2 \sigma^{2}}\right)
$$

where $\sigma^{2}$ is the variance of the noise.

$\mathrm{RR} \mathrm{n}^{\circ} 5130$ 


\section{Proposition 3.2.}

$$
E\left(\|n\|_{-1,2}^{2}\right)=C . E\left(\|n\|_{X}^{2}\right)
$$

where $C$ is a constant which depends only on the size of the image.

Proof: We begin by computing $E\left(\|n\|_{-1,2}^{2}\right)$.

We consider the functional:

$$
\inf _{u} F(u)
$$

where

$$
F(u)=\frac{1}{2}\|\nabla u\|_{Y}^{2}-(n, u)_{X}
$$

$F$ is convex and lsc (lower semi continuous). Hence (see [11] for instance) there exists $\tilde{u}$ solving problem (3.19). As in Subsection 3.3, we get:

$$
(\nabla \tilde{u}, \nabla h)_{Y}=(n, h)_{X} \forall h \text { with zero mean. }
$$

We denote by $\delta$ the image such that $\delta_{0,0}=1$, and $\delta_{i, j}=0$ otherwise. We denote by $W$ the solution of the problem:

$$
\inf _{u} \int_{\Omega}\left(\frac{1}{2}|\nabla u|^{2}-\delta u\right)
$$

and thus we have $(\nabla W, \nabla h)_{Y}=(\delta, h)_{X}$ for all $h$ with zero mean. We introduce a discrete convolution:

$$
f * g(x, y)=\sum_{i, j} f_{i, j} g_{x-i, y-j}
$$

It is easy to check that $\delta * f=f * \delta=f$ for all $f$ (in fact, $W$ is the Green function associated to problem (3.19)).

Let us now consider $u=n * W$; i.e. $u=\sum_{i, j} u_{i, j}$, with

$$
u_{i, j}(x, y)=n_{i, j} W_{x-i, y-j}
$$

$u$ is the solution of problem(3.19). If $h$ has zero mean, then $(\nabla u, \nabla h)_{Y}=$ $(n * \nabla W, \nabla h)_{Y}=(\nabla W, \bar{n} * \nabla h)_{Y}=(\delta, \bar{n} * h)_{X}=(n * \delta, h)_{X}=(n, h)_{X}$ (we have 
used the fact that $(n * a, b)_{X}=(a, \bar{n} * b)_{X}$ where $\left.\bar{n}_{i, j}=-n_{i, j}\right)$. From (3.11), we know that $\|n\|_{-1,2}=\|\nabla u\|_{2}$. We notice that $\nabla u=n * \nabla W$. Hence:

$$
\begin{aligned}
\sum_{i, j}\left|\nabla u_{i, j}\right|^{2} & =(n * \nabla W, n * \nabla W)_{Y} \\
& =(n, n * W)_{X} \\
& =\sum_{k, l} n_{k, l} n * W(k, l) \\
& =\sum_{k, l} n_{k, l} \sum_{i, j} n_{i, j} W_{k-i, l-j}
\end{aligned}
$$

We conclude that:

$$
\sum_{i, j}\left|\nabla u_{i, j}\right|^{2}=\sum_{i, j, k, l} n_{i, j} n_{k, l} W_{k-i, l-j}
$$

And thus:

$$
E\left(\sum_{i, j}\left|\nabla u_{i, j}\right|^{2}\right)=E\left(W_{k-i, l-j} n_{i, j} n_{k, l}\right)=W_{k-i, l-j} E\left(n_{i, j} n_{k, l}\right)
$$

As the $n_{i, j}$ are independant, we have $E\left(n_{i, j} n_{k, l}\right)=E\left(n_{i, j}\right) E\left(n_{k, l}\right)$ whenever $(i, j) \neq(k, l)$. Moreover, $E\left(n_{i, j}\right)=0$, and $E\left(n_{i, j}^{2}\right)=\sigma^{2}$. Hence:

$$
E\left(\sum_{i, j}\left|\nabla u_{i, j}\right|^{2}\right)=\sigma^{2} \sum_{i, j} W_{i-i, j-j}
$$

We thus get:

$$
E\left(\sum_{i, j}\left|\nabla u_{i, j}\right|^{2}\right)=N^{2} \sigma^{2} W_{0,0}
$$

We can also observe that:

$$
\sum_{i, j}\left|\nabla W_{i, j}\right|^{2}=(\nabla W, \nabla W)_{Y}=(\delta, W)_{X}=W_{0,0}
$$

So we can rewrite (3.28):

$$
E\left(\|\nabla u\|_{Y}^{2}\right)=N^{2} \sigma^{2}\|\nabla W\|_{Y}^{2}
$$

(and from Proposition 3.1 we know that $\|\nabla u\|_{Y}=\|n\|_{-1,2}$ )

$\mathrm{RR} \mathrm{n}^{\circ} 5130$ 
$L^{2}$ norm: We have:

$$
\|n\|_{X}^{2}=\sum_{i, j}\left(n_{i, j}\right)^{2}
$$

Hence

$$
E\left(\|n\|_{X}^{2}\right)=\sum_{i, j} E\left(\left(n_{i, j}\right)^{2}\right)
$$

And we get:

$$
E\left(\|n\|_{X}^{2}\right)=N^{2} \sigma^{2}
$$

We then get the result of Proposition 3.2 with $C=\|\nabla W\|_{Y}^{2}=W_{0,0}=$ $\|\delta\|_{-1,2}^{2}$.

This gives a simple test to know if an image can be a white gaussian noise or not. This result can also be useful when tuning the parameters of a denoisng algorithm using the $-1,2$ norm.

\section{Remark: Computation of the constant $C$ of Proposition 3.2 :}

Let us denote by $m_{\delta}$ the mean of $\delta$, and by $\dot{\delta}=\delta-m_{\delta}$.

From (3.21), we get that: $\Delta W=-\dot{\delta}$. So formally, we can write $W=$ $-\Delta^{-1}(\dot{\delta})$. We have shown that $C=\|\dot{\delta}\|_{-1,2}^{2}=\|\nabla W\|_{Y}^{2}$. We thus have $C=$ $\left(\nabla \Delta^{-1}(\dot{\delta}), \nabla \Delta^{-1}(\dot{\delta})\right)_{Y}=\left(-\dot{\delta}, \Delta^{-1}(\dot{\delta})\right)_{X}$. There remains to compute $\Delta^{-1}(\dot{\delta})$. This can easily be done with the Discrete Fourier Transform (DFT). We recall that the DFT of a given discrete image $(f(m, n))(0 \leq m \leq N-1$ and $0 \leq n \leq N-1)$ is given by $(0 \leq p \leq N-1$ and $0 \leq q \leq N-1)$ :

$$
\mathcal{F}(f)(p, q)=F(p, q)=\sum_{m=0}^{N-1} \sum_{n=0}^{N-1} f(m, n) e^{-j(2 \pi / N) p m} e^{-j(2 \pi / N) q n}
$$

and the inverse transform is:

$$
f(m, n)=\frac{1}{N^{2}} \sum_{p=0}^{N-1} \sum_{q=0}^{N-1} F(p, q) e^{j(2 \pi / N) p m} e^{j(2 \pi / N) q n}
$$


Table 3: Values of $\sqrt{C}=\|\dot{\delta}\|_{-1,2}=\|\nabla W\|_{X}$

\begin{tabular}{|c|c|c|c|c|c|c|c|c|c|c|c|}
\hline Image size & $16^{2}$ & $32^{2}$ & $64^{2}$ & $128^{2}$ & $256^{2}$ & $512^{2}$ & $1024^{2}$ & $2048^{2}$ & $4096^{2}$ & $8192^{2}$ & $16384^{2}$ \\
\hline$\sqrt{C}$ & 0.69 & 0.77 & 0.84 & 0.91 & 0.97 & 1.01 & 1.07 & 1.12 & 1.17 & 1.22 & 1.26 \\
\hline
\end{tabular}

Moreover, we also have $\|\mathcal{F}(f)\|_{X}^{2}=N^{2}\|f\|_{X}^{2}$ and $(\mathcal{F}(f), \mathcal{F}(g))_{X}=N^{2}(f, g)_{X}$.

Let us remark that $\mathcal{F}(\delta)(p, q)=1 \forall p$, and that $\mathcal{F}(\dot{\delta})(p, q)=0$ if $p=q=0$ and 1 otherwise.

We have $\Delta f(m, n)=f(m+1, n)+f(m-1, n)+f(m, n+1)+f(m, n-$ 1) $-4 f(m, n)$. Standard computations lead to:

$$
\mathcal{F}(\Delta f)(p, q)=2\left(\cos \left(\frac{2 \pi}{N} p\right)+\cos \left(\frac{2 \pi}{N} q\right)-2\right) \mathcal{F}(f)(p, q)
$$

We deduce that, if $f$ has zero mean, then for $(p, q) \neq(0,0)$

$$
\mathcal{F}\left(\Delta^{-1} f\right)(p, q)=\frac{1}{2\left(\cos \left(\frac{2 \pi}{N} p\right)+\cos \left(\frac{2 \pi}{N} q\right)-2\right)} \mathcal{F}(f)(p, q)
$$

Hence

$$
\mathcal{F}\left(\Delta^{-1} \dot{\delta}\right)(p, q)= \begin{cases}0 & \text { if } p=q=0 \\ \frac{1}{2\left(\cos \left(\frac{2 \pi}{N} p\right)+\cos \left(\frac{2 \pi}{N} q\right)-2\right)} & \text { otherwise }\end{cases}
$$

so that

$$
C=-\left(\dot{\delta}, \Delta^{-1} \dot{\delta}\right)_{X}=\frac{1}{N^{2}} \sum_{(p, q) \neq(0,0)} \frac{1}{2\left(2-\cos \left(\frac{2 \pi}{N} p\right)+\cos \left(\frac{2 \pi}{N} q\right)\right)}
$$

We have computed this way the constant $C$ for different image sizes (see Table 3).

Table 4 confirms the results of Table 3 . To fill in Table 4 , for each size (from $16^{*} 16$ to $512^{*} 512$ ), we have simulated 1000 images of white Gaussian

$\mathrm{RR} \mathrm{n}^{\circ} 5130$ 
Table 4: Numerical computations with 1000 images of white gaussian noise $n$ with standard deviation equal to 1 (for the $X$ norm and the $-1,2$ norm)

\begin{tabular}{|c|c|c|c|c|c|c|}
\hline Image size & $16^{2}$ & $32^{2}$ & $64^{2}$ & $128^{2}$ & $256^{2}$ & $512^{2}$ \\
\hline$E\left(\|n\|_{X}\right)$ & 16.0 & 32.0 & 64.0 & 128 & 256 & 512 \\
\hline$\sqrt{\operatorname{Var}\left(\|n\|_{X}\right)}$ & 0.71 & 0.69 & 0.72 & 0.73 & 0.73 & 0.70 \\
\hline$E\left(\|n\|_{-1,2}\right)$ & 11.2 & 24.7 & 54 & 116 & 247 & 523 \\
\hline$\sqrt{\operatorname{Var}\left(\|n\|_{-1,2}\right)}$ & 0.97 & 1.8 & 3.3 & 6.0 & 12 & 22 \\
\hline$\frac{E\left(\|n\|_{X}\right)}{E\left(\|n\|_{-1,2}\right)}$ & 0.70 & 0.77 & 0.84 & 0.91 & 0.96 & 1.02 \\
\hline
\end{tabular}

Table 5: Numerical computations with 1000 images of white gaussian noise $n$ with standard deviation equal to 1 (for the $T V$ norm and the $E$ norm)

\begin{tabular}{|c|c|c|c|c|c|c|}
\hline Image size & $16^{2}$ & $32^{2}$ & $64^{2}$ & $128^{2}$ & $256^{2}$ & $512^{2}$ \\
\hline$E(J(n))$ & 392 & 1670 & 6920 & 28100 & 113000 & 455000 \\
\hline$\sqrt{\operatorname{Var}(J(n))}$ & 20 & 43 & 90 & 180 & 360 & 690 \\
\hline$E\left(\|n\|_{E}\right)$ & 2.81 & 3.59 & 3.63 & 3.98 & 4.30 & 4.59 \\
\hline$\sqrt{\operatorname{Var}\left(\|n\|_{E}\right)}$ & 0.40 & 0.34 & 0.32 & 0.32 & 0.28 & 0.26 \\
\hline
\end{tabular}

noise with standard deviation $\sigma$ equal to 1.0 (to normalize our experiment). Then we have computed the $X$ norm and the $-1,2$ norm of each image. We have computed the experimental mean and and also the experimental standard deviation. The values of $\frac{E\left(\|n\|_{X}\right)}{E\left(\|n\|_{-1,2}\right)}$ of Table 4 fit the values of $\sqrt{C}$ of Table 3 , as expected from Proposition 3.2. But the experimental standard deviations of these norms do not have the same behaviour: the standard deviation of the $X$ remains almost constant, whereas the standard deviation of the $-1,2$ norm seems to be an increasing function of the size of the image. Nevertheless, the standard deviation of the $-1,2$ norm seems to increase slower then its mean, and as soon as the size of the image is large enough, the standard deviation remains small with repect to the mean (which can therefore be considered as a quite good estimate of the $-1,2$ norm). 
Table 5 shows the results we get for the same experiment with the $T V$ and the $E$ norm (we recall that $J(f)=\|f\|_{T V}$ ).

\subsection{An algorithm to solve the Osher-Solé-Vese model}

We come back here to the model proposed by Osher-Solé-Vese in [17]. We recall that the considered problem is:

$$
\inf _{u}\left(J(u)+\frac{1}{2 \lambda}\|f-u\|_{-1,2}^{2}\right)
$$

The following result holds:

Proposition 3.3. If $\hat{u}$ is a solution of problem (3.40), then $\hat{v}=\Delta^{-1}\left(\frac{\hat{u}-f}{\lambda}\right)$ is a solution of the dual problem:

$$
\inf _{v}\left(\frac{\lambda}{2}\|\nabla v\|_{Y}^{2}-(f, v)_{X}+J^{*}(v)\right)
$$

Proof: There are many ways to show this (see [10]). For instance, one may reproduce the proof of Proposition 2.3 given in [6]. Thanks to Proposition 3.1, we have $\|f-u\|_{-1,2}^{2}=\left(-f+u, \Delta^{-1}(f-u)\right)_{X}$. If $u$ is a minimiser of (3.40), then: $0 \in-\Delta^{-1}\left(\frac{u-f}{\lambda}\right)+\partial J(u)$, i.e.: $\Delta^{-1}\left(\frac{u-f}{\lambda}\right) \in \partial J(u)$. Hence $u \in \partial J^{*}\left(\Delta^{-1}\left(\frac{u-f}{\lambda}\right)\right)$, i.e.: $0 \in \frac{f-u}{\lambda}-\frac{f}{\lambda}+\frac{1}{\lambda} \partial J^{*}\left(\Delta^{-1}\left(\frac{u-f}{\lambda}\right)\right)$. We then set $v=\Delta^{-1}\left(\frac{u-f}{\lambda}\right)$, and we get: $0 \in-\Delta v-\frac{f}{\lambda}+\frac{1}{\lambda} \partial J^{*}(v)$. $v$ is thus a solution of problem (3.41).

Algorithm: Thanks to Proposition 3.3, we have $\hat{u}=f+\lambda \Delta \hat{v}$. Hence, to compute $\hat{u}$, we just need to compute $\hat{v}$ solution of problem (3.41). As $J^{*}$ is given by (2.15), $\hat{v}$ is the solution of the following problem:

$$
\min \left\{\frac{\lambda}{2}\|\nabla \operatorname{div}(p)\|_{Y}^{2}+(\nabla f, p)_{Y}: p /\left|p_{i, j}\right| \leq 1 \forall i, j=1, \ldots, N\right\}
$$

$\mathrm{RR} \mathrm{n}^{\circ} 5130$ 
By using the same arguments as in [6], one sees that this problem can be numerically solved by a fixed point method.

$$
p^{0}=0
$$

and

$$
p_{i, j}^{n+1}=\frac{p_{i, j}^{n}-\tau\left(\nabla\left(\Delta \operatorname{div}\left(p^{n}\right)+f / \lambda\right)\right)_{i, j}}{1+\tau\left|\left(\nabla\left(\Delta \operatorname{div}\left(p^{n}\right)+f / \lambda\right)\right)_{i, j}\right|}
$$

And as in [6], we show that if $\tau$ is small enough, $f+\lambda \Delta \operatorname{div} p^{n} \rightarrow \hat{u}$ as $n \rightarrow \infty$.

Numerical results Figure 7 is to be compared with Figure 1. We can see that as a denoising algorithm, the OSV model seems to outperforms both the ROF model and Meyer model.

Figure 6 shows a comparison between the OSV model and the algorithm of [4]. As a decomposition algorithm (that is as an algorithm which splits a given image into a geometrical component and an oscillatory component), the

algorithm of [4] seems to outperform the OSV model.

Remark: In the OSV model (3.40), this is not the norm but the square of the norm which is minimized for the oscillatory component (but this is equivalent up to a a change of parameter $\lambda$ ).

\section{Minimization of dual norms}

\subsection{Some convex analysis}

Proposition 4.1. Let $F$ be a convex function. Then the two following statements are equivalent:

1. $\tilde{u}$ is a solution of

$$
\min _{u}\left(F(u)+\frac{1}{2 \lambda}\|f-u\|_{X}^{2}\right)
$$

2. $\tilde{w}=f-\tilde{u}$ is a solution of

$$
\min _{w}\left(F^{*}(w / \lambda)+\frac{1}{2 \lambda}\|f-w\|_{X}^{2}\right)
$$



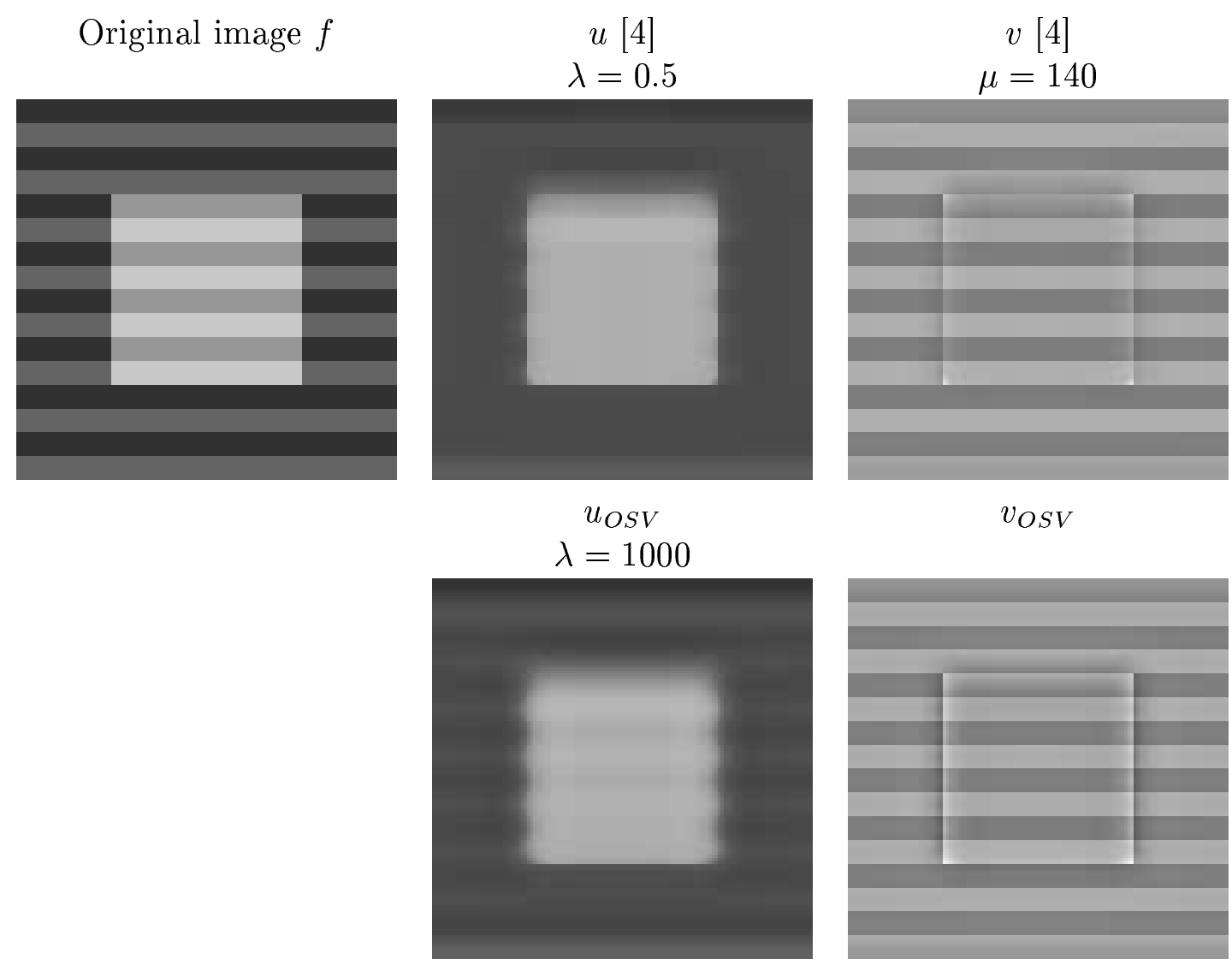

$v_{O S V}$

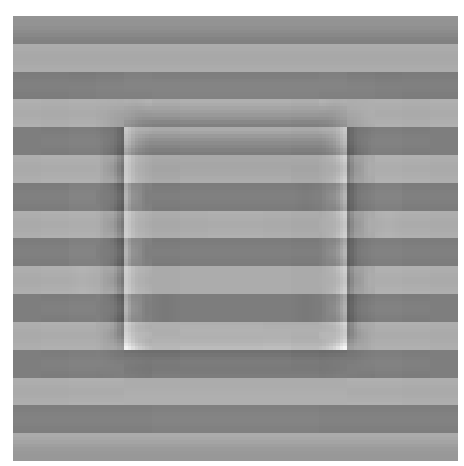

Figure 6: Decomposition (the parameters are tuned so that both $v_{O S V}$ and the $v$ component got with the algorithm of [4] have the same $L^{2}$ norm)

$\mathrm{RR} \mathrm{n}^{\circ} 5130$ 


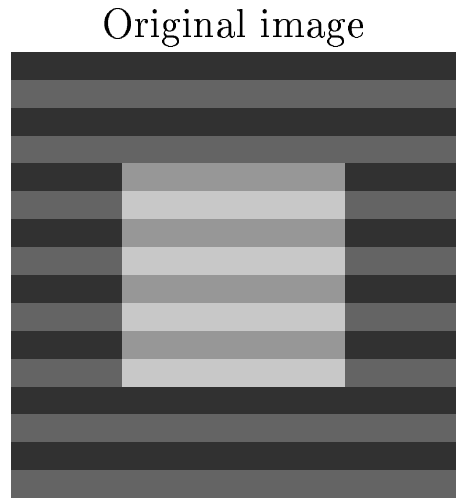

Restored image

$$
\lambda=70.0
$$
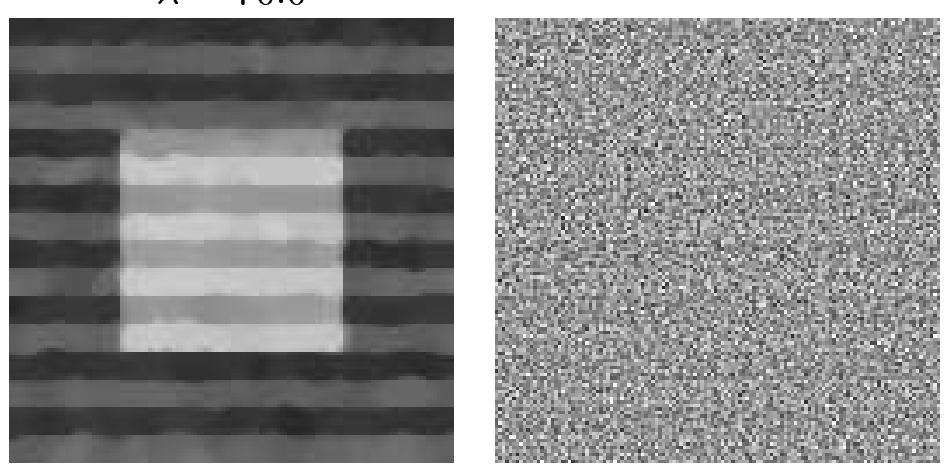

Figure 7: Denoising stripes with the Osher-Sole-Vese model

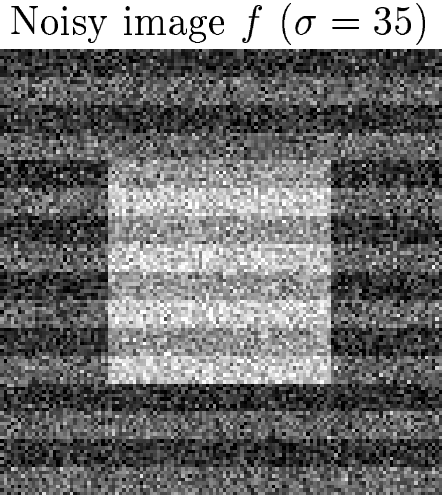

Noise 
Moreover, if $F$ is 1 homogeneous (this is the case when $F$ is a semi norm), then $F^{*}$ is the indicator function of a closed convex set $K$.

\section{Examples:}

1. If $F(u)=J(u)($ defined by $(1.1))$, then

$$
F^{*}(w)=\chi_{\left\{v /\|v\|_{G} \leq 1\right\}}(w)
$$

2. If $F(u)=\|u\|_{G}($ defined by (1.3)), then

$$
F^{*}(w)=\chi_{\{v / J(v) \leq 1\}}(w)
$$

3. If $F(u)=\|u\|_{-1, p}$, then

$$
F^{*}(w)=\chi_{\left\{v /\|\nabla v\|_{q} \leq 1\right\}}(w)
$$

4. If $F(u)=\|u\|_{\dot{B}_{1,1}^{1}}$, then

$$
F^{*}(w)=\chi_{\left\{v /\|v\|_{B_{-1, \infty}^{\infty}}^{\infty} \leq 1\right\}}(w)
$$

Proof of Proposition 4.1 This is a standard duality result for variational problems [10].

$\tilde{u}$ is a solution of

$$
\min _{u}\left(F(u)+\frac{1}{2 \lambda}\|f-u\|_{X}^{2}\right)
$$

if and only if $0 \in(1 / \lambda)(\tilde{u}-f)+\partial F(\tilde{u})$, i.e if and only if $(1 / \lambda)(f-\tilde{u}) \in \partial F(\tilde{u})$, which is equivalent to $\tilde{u} \in \partial F^{*}\left(\frac{f-\tilde{u}}{\lambda}\right)$, i.e

$$
0 \in f-\tilde{u}-f+\partial F^{*}\left(\frac{f-\tilde{u}}{\lambda}\right)
$$

which precisely means that $\tilde{w}=f-\tilde{u}$ is a solution of

$$
\min _{w}\left(\lambda F^{*}(w / \lambda)+\frac{1}{2}\|f-w\|_{X}^{2}\right)
$$

$\mathrm{RR} \mathrm{n}^{\circ} 5130$ 


\subsection{Application to $u+v$ models}

Let us now consider a functional of the form:

$$
\min _{u, v}\left(F(u)+\frac{1}{2 \lambda}\|f-u-v\|_{X}^{2}+L(v)\right)
$$

where $F$ and $L$ are two convex functions. A natural algorithm to minimize such a functional consists in minimizing succesively with respect to each variable, that is to solve sucessively the two following problems:

$$
\begin{aligned}
& \min _{u}\left(F(u)+\frac{1}{2}\|f-u-v\|_{X}^{2}\right) \\
& \min _{v}\left(\frac{1}{2}\|f-u-v\|_{X}^{2}+L(v)\right)
\end{aligned}
$$

Let us assume that $F(u)=J(u)$ defined by (1.1). We will consider several possibilities for the function $L$ :

$$
L(v)=\mu\|v\|_{-1, p}
$$

Comments: Using Proposition 4.1, one sees that $\tilde{v}$ is a solution of problem (4.12) if and only if $\tilde{w}=f-u-\tilde{v}$ is a solution of the problem:

$$
\min _{w}\left(L^{*}(w)+\frac{1}{2}\|f-u-w\|_{X}^{2}\right)
$$

- If $p=2$ in (4.13), then using (4.5) we see that the minimization of (4.12) amounts to a Tikhonov regularization with $H^{1}$ penalization (and one knows that such a regularization is not a good choice in image processing since it does not preserve edges).

- If $p=1$ in (4.13), then using (4.5) we see that the minimization of (4.12) amounts to a Lipschitz regularization of the gradient: this is not a good choice in image processing either.

- More generally, if $p \in[1,+\infty)$, we see that such a minimization smoothes too much the geometrical part of the image. 
Conclusion If one wants to minimize a functional like (4.10) to capture oscillating patterns, then such considerations are in favor of the use of the $G$ norm, as advocated in [16]. Even if such a norm is much more complicated to compute (especially comparing with the $-1,2$ norm), it seems worth to use it.

\section{A new $u+v$ algorithm}

\subsection{Principle}

In [16], the author proposes to minimize the following functional:

$$
\inf _{u+v=f}\left(J(u)+\beta\|v\|_{E}\right)
$$

Due to the $E=\dot{B}_{-1, \infty}^{\infty}$ norm, this problem cannot be solved directly. We propose instead to minimize the following functional:

$$
\inf _{(u, v) \in X^{2}}\left(J(u)+B^{*}(v / \delta)+\frac{1}{2 \lambda}\|f-u-v\|_{X}^{2}\right)
$$

where

$$
B(w)=\|w\|_{\dot{B}_{1,1}^{1}}
$$

and therefore

$$
B^{*}(w / \delta)=\chi\left\{\|w\|_{E} \leq \delta\right\}
$$

Furthermore, let us denote by

$$
\delta B_{E}=B_{E(\delta)}=\left\{w /\|w\|_{E} \leq \delta\right\}
$$

When $\lambda$ goes to 0 , one can show that solving problem (5.16) gives a solution for (5.15) (using the same arguments as in [4]).

To minimize (5.16), we consider the two following problems:

- $v$ being fixed, we search for $u$ as a solution of:

$$
\inf _{u \in X}\left(J(u)+\frac{1}{2 \lambda}\|f-u-v\|_{X}^{2}\right)
$$

$\mathrm{RR} \mathrm{n}^{\circ} 5130$ 
- $u$ being fixed, we search for $v$ as a solution of:

$$
\inf _{v \in \delta B_{E}}\|f-u-v\|_{X}^{2}
$$

From Proposition 2.3, we know that the solution of (5.20) is given by: $\hat{u}=f-v-P_{\lambda B_{G}}(f-v)$. And the solution of (5.21) is given by: $\hat{v}=P_{\delta B_{E}}(f-u)$.

To compute this last projection, we consider the dual problem of (5.21):

$$
\inf _{w \in X}\left(\frac{1}{2}\|f-u-w\|_{X}^{2}+\delta\|w\|_{\dot{B}_{1,1}^{1}}\right)
$$

The solution of this problem $\hat{w}$ is obtained by a wavelet soft-thresholding algorithm with threshold $2 \delta$ (see [8]). We write $\hat{w}=W S T(f-u, 2 \delta)$. And from proposition 4.1 , we see that $\hat{v}=f-u-\hat{w}$.

\subsubsection{Algorithm}

1. Initialization:

$$
u_{0}=v_{0}=0
$$

2. Iterations:

$$
\begin{gathered}
v_{n+1}=P_{\delta B_{E}}\left(f-u_{n}\right)=f-u_{n}-W S T\left(f-u_{n}, 2 \delta\right) \\
u_{n+1}=f-v_{n+1}-P_{\lambda B_{G}}\left(f-v_{n+1}\right)
\end{gathered}
$$

3. Stopping test: we stop if

$$
\max \left(\left|u_{n+1}-u_{n}\right|,\left|v_{n+1}-v_{n}\right|\right) \leq \epsilon
$$

\subsection{Mathematical analysis}

We follow here [4]. The proofs of the results stated in this subsection are the same as in [4].

The first point we state is the convergence of the proposed algorithm. 
Proposition 5.2. The sequence $\left(u_{n}, v_{n}\right)$ built in (5.23)-(5.26) converges to $\left(u_{0}, v_{0}\right) \in X \times X$ the unique solution of problem (5.16).

To see the link between (5.15) and (5.16), we introduce the following problem:

$$
\inf _{u+v=f}\left(J(u)+B^{*}(v / \delta)\right)
$$

The next result states the link between (5.15) and (5.27)

Proposition 5.3. Let us fix $\beta>0$ in problem (5.15). Let $(\hat{u}, \hat{v})$ a solution of problem (5.15). We fix $\delta=\|\hat{v}\|_{E}$ in (5.27). Then:

- $(\hat{u}, \hat{v})$ is also a solution of problem (5.27).

- Conversely, any solution $(\tilde{u}, \tilde{v})$ of (5.27) (with $\left.\delta=\|\hat{v}\|_{E}\right)$ is a solution of (5.15).

To close the link between (5.15) and (5.16), we have to check what happens when $\lambda$ goes to 0 in problem (5.16).

Proposition 5.4. Let us fix $\beta>0$ in (5.15). Set $\delta=\|\hat{v}\|_{E}$ in (5.16) and (5.27). Let us denote by $\left(u_{\lambda_{n}}, v_{\lambda_{n}}\right)$ the solution of problem (5.16) (with $\left.\lambda=\lambda_{n}\right)$. Then, when $\lambda_{n}$ goes to 0 , any cluster point of $\left(u_{\lambda_{n}}, v_{\lambda_{n}}\right)$ is a solution of problem (5.27).

\subsection{Numerical results and comments}

Choice of the parameters: The parameter $\lambda$ controls the $L^{2}$ norm of the residual $f-u-v$. The smaller it is, the smaller the norm of the residual becomes. In practice, we just need to set $\lambda$ small (in our experiments, we have used $\lambda \leq 1$ : the $L^{\infty}$ norm of the residual is then smaller than 1.5 (and the gray values of our images go from 0 to 255$)$ ).

The parameter $\delta$ represents the $E$ norm of the $v$ component. Having a closer look at the algorithm, we see that $2 \delta$ is the threshold which is used in the wavelet thresholding. In the case of denoising, we can then use the classical threshold $\sigma \sqrt{2 \log \left(N^{2}\right)}$ where $N^{2}$ is the size of the image $[9,13,16]$.

We therefore choose $\delta=\frac{\sigma \sqrt{2 \log \left(N^{2}\right)}}{2}$. In fact, to get better results, we have

$\mathrm{RR} \mathrm{n}^{\circ} 5130$ 


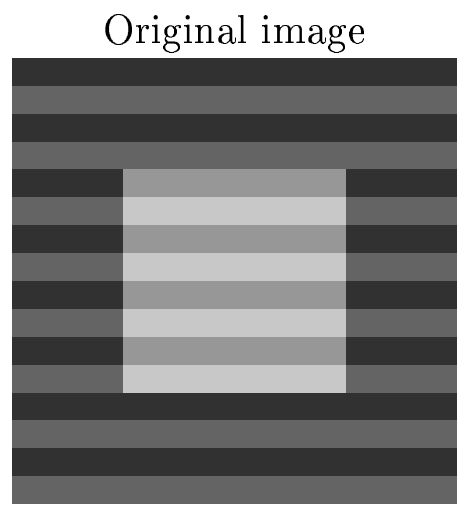

Restored image

$\lambda=0.5$
Noisy image $f(\sigma=35)$

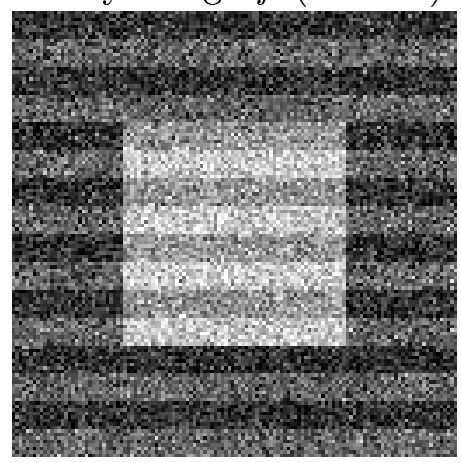

Noise

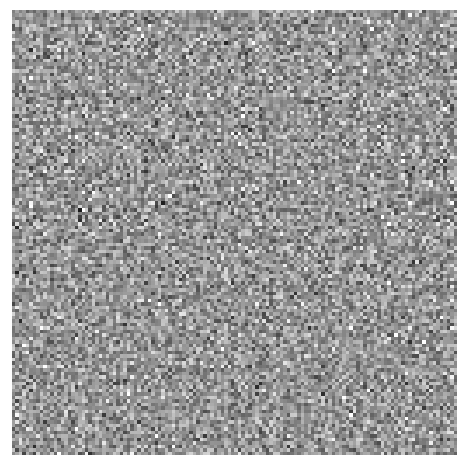

Figure 8: Denoising stripes

introduced a weighting parameter $\eta$, and we set $\delta=\eta \frac{\sigma \sqrt{2 \log \left(N^{2}\right)}}{2}$. In practice, $\eta$ is equal to 1 or slightly smaller.

Comments: This new algorithm appears to be a very good denoising algorithm for textured images. Indeed, it simultaneously minimizes the total variation of the restored image and a Besov norm (which amounts to a wavelet shrinkage). It benefits from the advantages of both methods. One sees on Figure 9 that the restored image has been more regularized with this new model than with the classical wavelet thresholding. Figure 8 is to be compared with Figure 1 and Figure 7 . In this case, our new denoising algorithm clearly outper- 


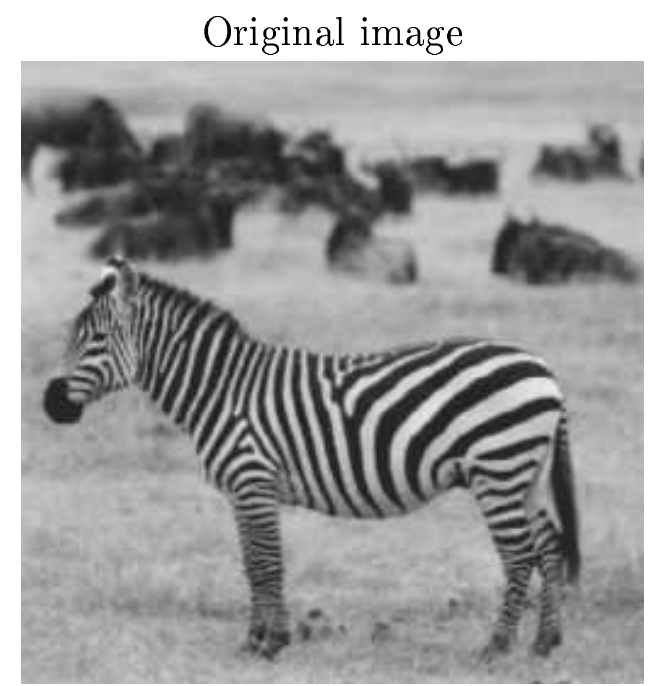

Restoration

$(\lambda=0.5, \eta=0.7$, Daub8)

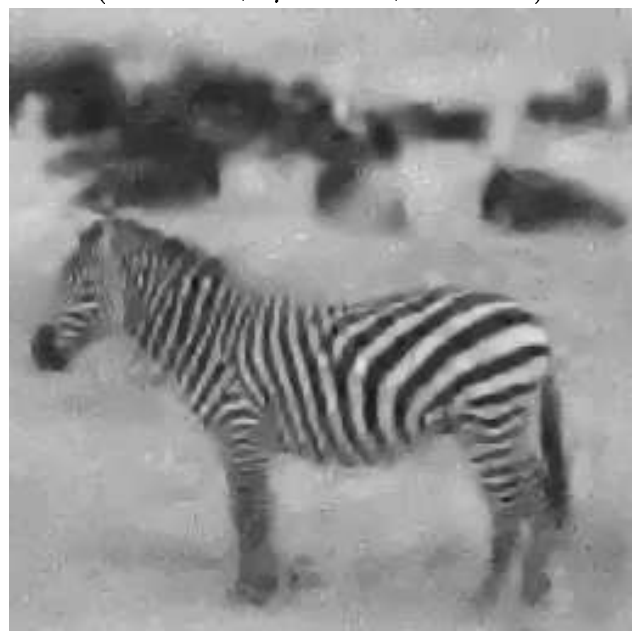

Noisy image $(\sigma=35)$

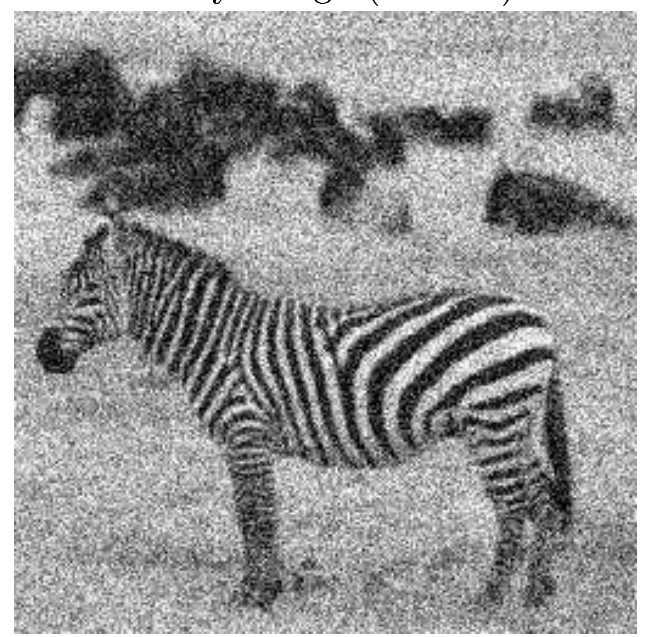

Wavelet thresholding

$(\eta=0.7$, Daub8)

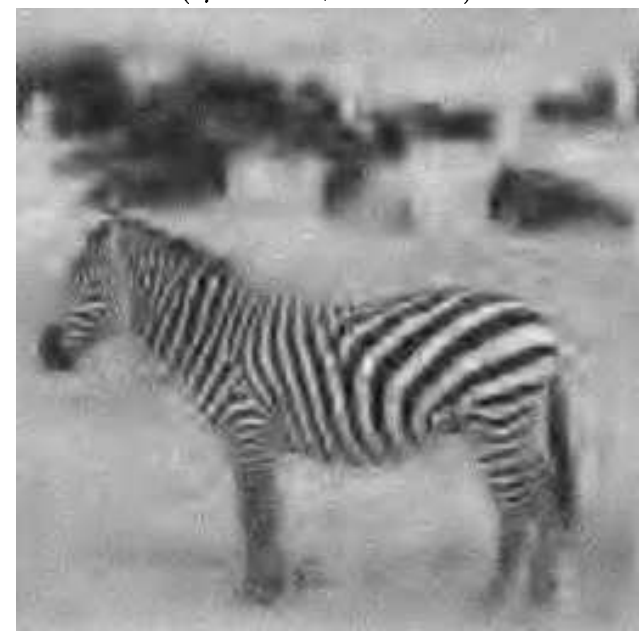

Figure 9: Zebra image

$\mathrm{RR} \mathrm{n}^{\circ} 5130$ 


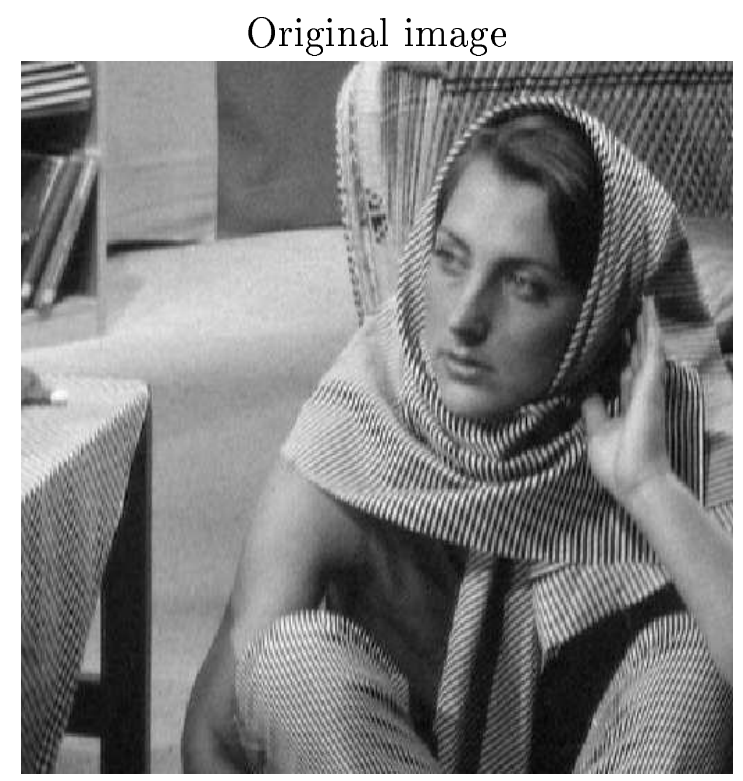

Restoration

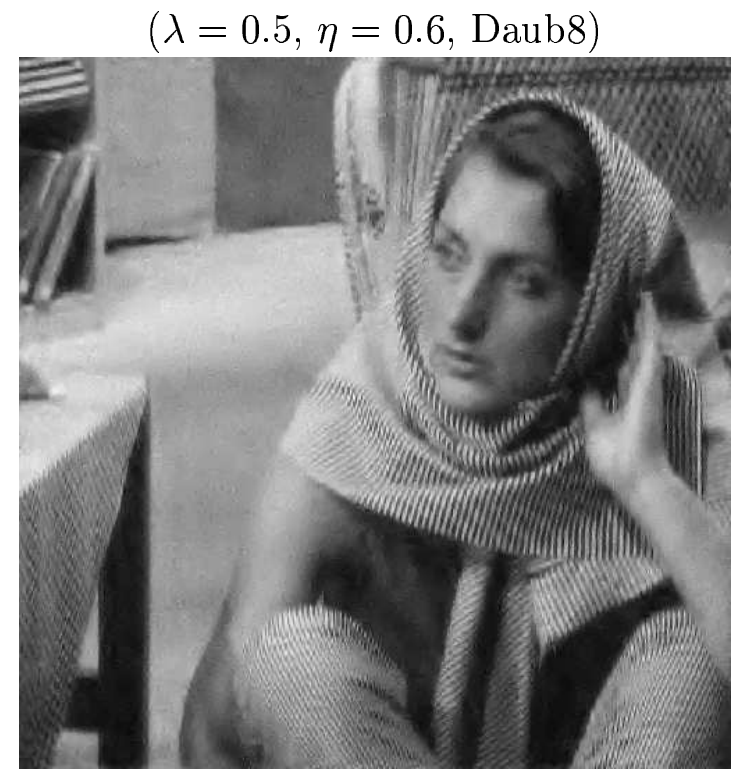

Noisy image $(\sigma=20)$

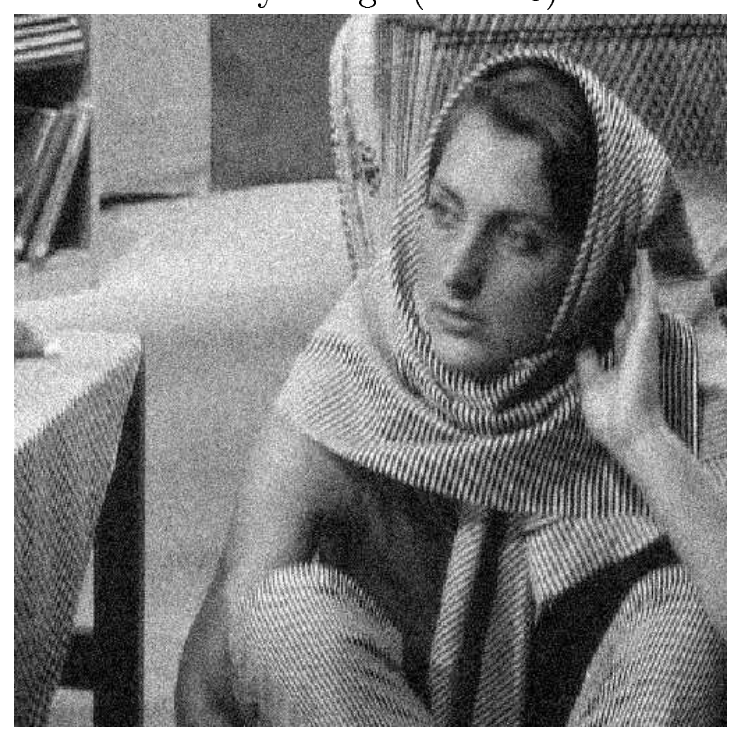

Restoration with

the Rudin-Osher-Fatemi model

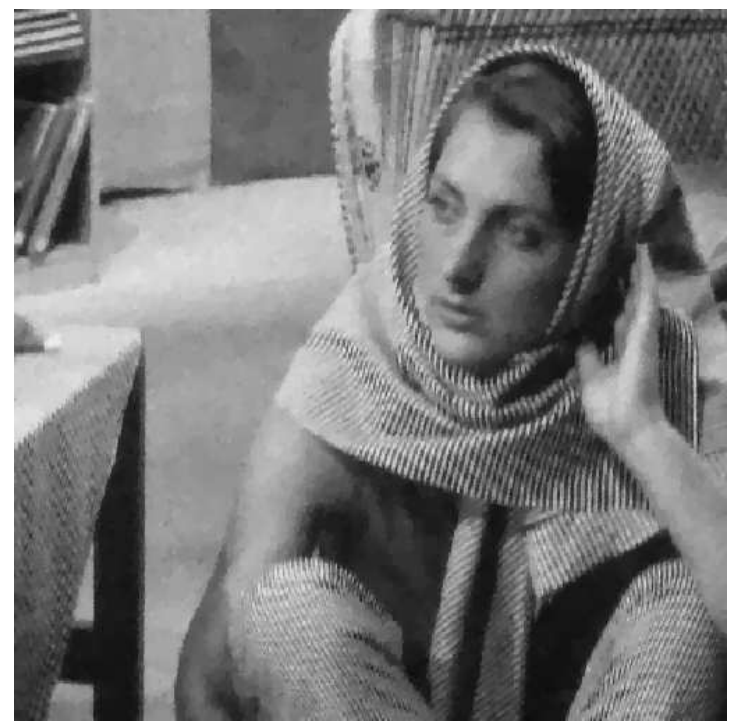

Figure 10: Barbara 

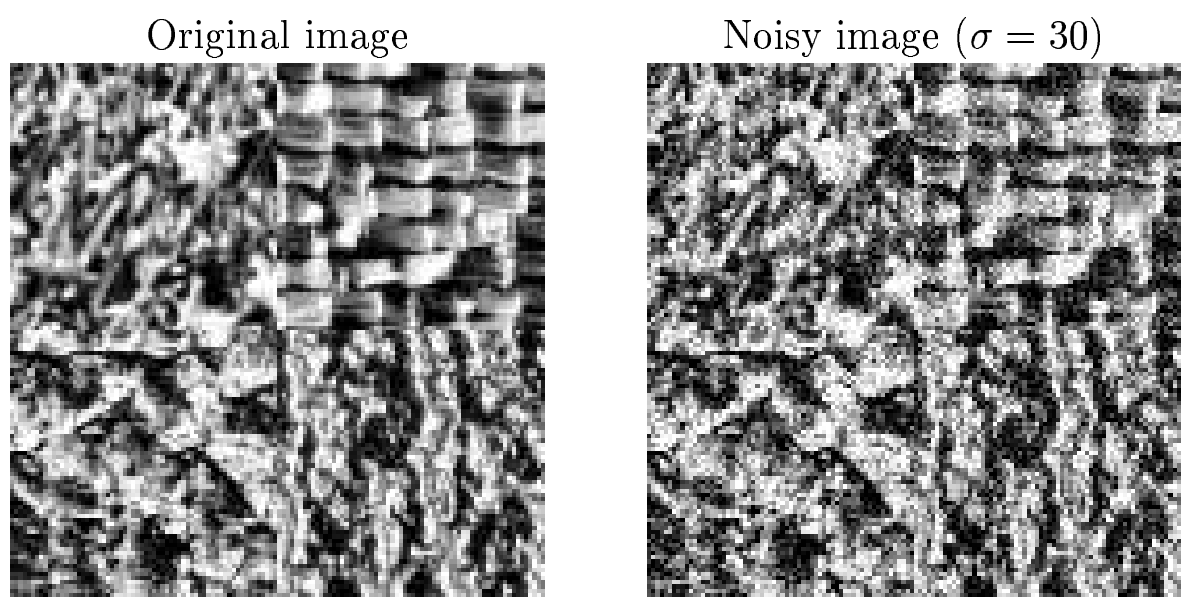

Restoration

Restoration with the algorithm of [4]

$$
(\lambda=0.5, \eta=0.6, \text { Daub8 })
$$

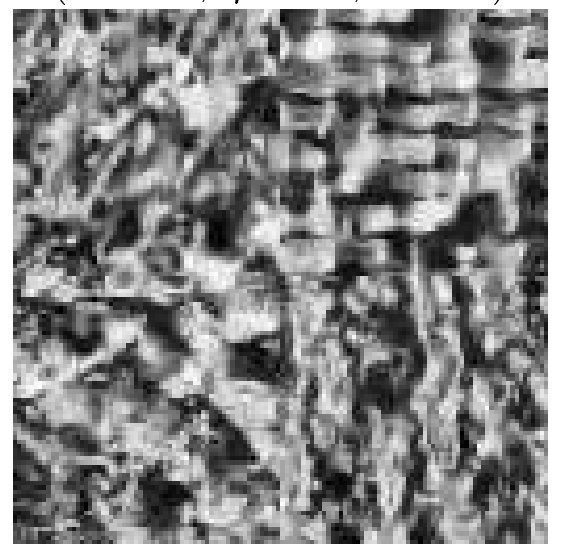

$$
(\lambda=0.5, \mu=0.75)
$$

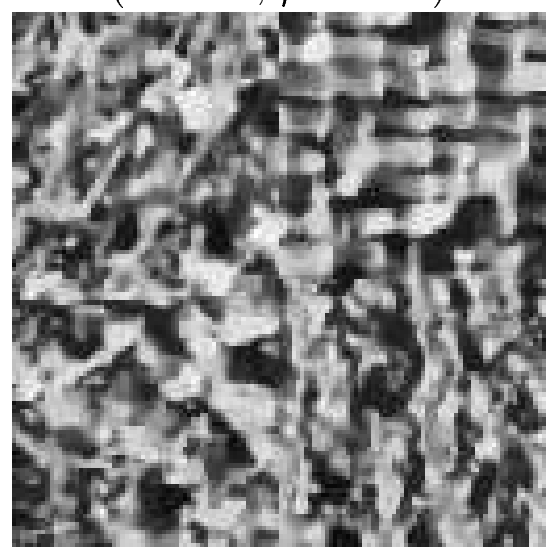

Figure 11: Texture image 
forms the ROF model [18] or the algorithm of [4]. And it also appears slightly better than the OSV model [17]. One sees on Figure 10 that the texture has been more preserved than with the Rudin-Osher-Fatemi model [18,6]. And on Figure 11, one sees that the small stucture of textures are better preserved than with the algorithm of [4]. This is why this model is particularly well suited to denoise textured images.

In $[13,12]$, the author also proposes to simultaneously combine the advantage of the total variation minimization and wavelet thresholding. He introduces a general framework which contains both these two approaches. Moreover, he can deal with other dictionaries than wavelet bases. But the numerical approach is completely different. He solves a penalization problem which is more complicated.

Therefore, to construct a model which would decompose an image into three components, a first one containing the geometrical information of the image, a second one containing the texture, and a last one containing the noise, it is then natural to consider the three following norms: the total variation, the $G$ norm, and the $E$ norm.

\section{6. $u+v+w$ model}

\subsection{Presentation}

We propose to minimize the following functional:

$$
\inf _{(u, v, w) \in X^{3}} F(u, v, w)
$$

where

$$
F(u, v, w)=J(u)+J^{*}\left(\frac{v}{\mu}\right)+B^{*}\left(\frac{w}{\delta}\right)+\frac{1}{2 \lambda}\|f-u-v-w\|_{X}^{2}
$$

Proposition 6.1. (6.1) admits a solution $(\hat{u}, \hat{v}, \hat{w})$. Moreover, $\hat{u}$ and $\hat{v}+\hat{w}$ are unique.

Proof: We split the proof into two steps:

Step 1: Existence 
1. We first remark that the set $X \times \mu B_{G} \times \delta B_{E}$ is convex, and then that $F$ is convex on $X \times \mu B_{G} \times \delta B_{E}$. We thus deduce that $F$ is convex on $X \times X \times X$.

2. It is immediate to see that $F$ is continuous on $X \times \mu B_{G} \times \delta B_{E}$. We then deduce that $F$ is lower semi-continuous on $X \times X \times X$.

3. Let $(u, v, w) \in X \times \mu B_{G} \times \delta B_{E}$. We have $\|v\|_{G} \leq \mu$. Moreover, since $X$ is of finite dimension, there exists $g \in X$ such that $v=\operatorname{div}(g)$ and $\|g\|_{L^{\infty}}=\|v\|_{G} \leq \mu$. We deduce from (2.8) that $\left(N^{2}\right.$ is the size of the image):

$$
\|v\|_{X} \leq 4 \mu N^{2}
$$

Since $w \in \delta B_{E}$, and since $X$ is of finite dimension, we get that there exists $C>0$ depending only on $N$ and $\delta$ such that:

$$
\|w\|_{X} \leq C
$$

We recall that $X \times X \times X$ is endowed with the Euclidean norm.

$$
\|(u, v, w)\|_{X \times X \times X}=\sqrt{\|u\|_{X}^{2}+\|v\|_{X}^{2}+\|w\|_{X}^{2}}
$$

Thus, if $\|(u, v, w)\|_{X \times X} \rightarrow+\infty$, then we get from (6.3) and (6.4) that $\|u\|_{X} \rightarrow+\infty$. We therefore deduce, since $f$ is fixed, and since (6.3) and (6.4) hold, that $\|f-u-v-w\|_{X}^{3} \rightarrow+\infty$. And since $F(u, v, w) \geq$ $\frac{1}{2 \lambda}\|f-u-v-w\|_{X}^{2}$, we get $F(u, v, w) \rightarrow+\infty$. Hence we deduce that $F$ is coercive on $X \times \mu B_{G} \times \delta B_{E}$. We therefore conclude that $F$ is coercive on $X \times X \times X$.

We deduce the existence of a minimizer $(\hat{u}, \hat{v}, \hat{w})$.

Step 2: Uniqueness of $\hat{u}$ and $\hat{v}+\hat{w}$ :

To get this result, we first remark that $F$ is strictly convex on $X \times \mu B_{G} \times \delta B_{E}$, as the sum of a convex function and of a strictly convex function, except in the direction $(u, v,-u-v)$. Hence it suffices to check that if $(\hat{u}, \hat{v}, \hat{w})$ is a minimizer of $F$ then for $t \neq 0,(\hat{u}+t \hat{u}, \hat{v}+t \hat{v}, \hat{w}-t(\hat{u}+\hat{v}))$ is not a minimizer of $F$. And since $F$ is convex, we can assume $|t|<1$. The result is obvious if 
$\hat{v}+t \hat{v} \in X \backslash \mu B_{G}$ or $\hat{w}-t(\hat{u}+\hat{v}) \in X \backslash \delta B_{E}$. Let us show that if $\hat{v}+t \hat{v} \in \mu B_{G}$ and $\hat{w}-t(\hat{u}+\hat{v}) \in \delta B_{E}$, then the result is still true. Indeed, we have in this case (since $|t|<1$ ):

$$
F(\hat{u}+t \hat{u}, \hat{v}+t \hat{v}, \hat{w}-t(\hat{u}+\hat{v}))=F(\hat{u}, \hat{v}, \hat{w})+t J(\hat{u})
$$

By contradiction, let us assume that there exists $\hat{t} \neq 0$ such that $\hat{w}-\hat{t}(\hat{u}+\hat{v}) \in$ $\delta B_{E}$.

$$
F(\hat{u}+\hat{t} \hat{u}, \hat{v}+\hat{t} \hat{v}, \hat{w}-\hat{t}(\hat{u}+\hat{v})) \leq F(\hat{u}, \hat{v}, \hat{w})
$$

As $(\hat{u}, \hat{v}, \hat{w})$ minimizes $F,(6.7)$ is an equality. From (6.6), we deduce that $J(\hat{u})=0($ since $\hat{t} \neq 0)$. There exists therefore $\gamma \in \mathbb{R}$ such that for all $(i, j)$, $\hat{u}_{i, j}=\gamma$.

1. If $\gamma=0$, then $\hat{u}=0$. Thus $(\hat{u}+\hat{t} \hat{u}, \hat{v}+\hat{t} \hat{v}, \hat{w}-\hat{t}(\hat{u}+\hat{v}))=(\hat{u}, \hat{v}+\hat{t} \hat{v}, \hat{w}-\hat{t} \hat{v})$.

2. If $\gamma \neq 0$, then $\hat{w}-\hat{t}(\hat{u}+\hat{v})$ cannot belong to $\delta B_{E}$ since its mean is not 0 . This contradicts our assumption.

To solve (6.1), we consider the three following problems:

- $v$ and $w$ being fixed, we search for $u$ as a solution of:

$$
\inf _{u \in X}\left(J(u)+\frac{1}{2 \lambda}\|f-u-v-w\|_{X}^{2}\right)
$$

- $u$ and $w$ being fixed, we search for $v$ as a solution of:

$$
\inf _{v \in \mu B_{G}}\|f-u-v-w\|_{X}^{2}
$$

- $u$ and $v$ being fixed, we search for $w$ as a solution of:

$$
\inf _{w \in \delta B_{E}}\|f-u-v-w\|_{X}^{2}
$$

From Proposition 2.3, we know that the solution of (6.8) is given by: $\hat{u}=f-v-w-P_{\lambda B_{G}}(f-v-w)$. The solution of (6.9) is simply given by: $\hat{v}=P_{\mu B_{G}}(f-u-w)$. And remembering Section 5, the solution of (6.10) is given by: $\hat{w}=P_{\delta B_{E}}(f-u-v)=f-u-v-W S T(f-u-v, 2 \delta)$, where $W S T(f-u-v, 2 \delta)$ stands for the wavelet soft-thresholding of $f-u-v$ with threshold $2 \delta$. 


\subsection{Algorithm}

\section{Algorithm 1:}

1. Initialization:

$$
u_{0}=v_{0}=w_{0}=0
$$

2. Iterations:

$$
\begin{gathered}
w_{n+1}=P_{\delta B_{E}}\left(f-u_{n}-v_{n}\right)=f-u_{n}-v_{n}-W S T\left(f-u_{n}-v_{n}, 2 \delta\right) \\
v_{n+1}=P_{\mu B_{G}}\left(f-u_{n}-w_{n+1}\right) \\
u_{n+1}=f-v_{n+1}-w_{n+1}-P_{\lambda B_{G}}\left(f-v_{n+1}-w_{n+1}\right)
\end{gathered}
$$

3. Stopping test: we stop if

$$
\max \left(\left|u_{n+1}-u_{n}\right|,\left|v_{n+1}+w_{n+1}-v_{n}-w_{n}\right|\right) \leq \epsilon
$$

4. We thus get $\hat{u}=\lim _{n}\left(u_{n}\right)$. Then, iterations:

$$
\begin{gathered}
w_{n+1}=P_{\delta B_{E}}\left(f-\hat{u}-v_{n}\right)=f-\hat{u}-v_{n}-W S T\left(f-\hat{u}-v_{n}, 2 \delta\right) \\
v_{n+1}=P_{\mu B_{G}}\left(f-\hat{u}-w_{n+1}\right)
\end{gathered}
$$

5. Stopping test: we stop if

$$
\max \left(\left|v_{n+1}-v_{n}\right|,\left|w_{n+1}-w_{n}\right|\right) \leq \epsilon
$$

In practice, we rather use the following algorithm (numerically, it seems to give the same results).

$\mathrm{RR} \mathrm{n}^{\circ} 5130$ 


\section{Algorithm 2:}

1. Initialization:

$$
u_{0}=v_{0}=w_{0}=0
$$

2. Iterations:

$$
\begin{gathered}
w_{n+1}=P_{\delta B_{E}}\left(f-u_{n}-v_{n}\right)=f-u_{n}-v_{n}-W S T\left(f-u_{n}-v_{n}, 2 \delta\right) \\
v_{n+1}=P_{\mu B_{G}}\left(f-u_{n}-w_{n+1}\right) \\
u_{n+1}=f-v_{n+1}-w_{n+1}-P_{\lambda B_{G}}\left(f-v_{n+1}-w_{n+1}\right)
\end{gathered}
$$

3. Stopping test: we stop if

$$
\max \left(\left|u_{n+1}-u_{n}\right|,\left|v_{n+1}-v_{n}\right|,\left|w_{n+1}-w_{n}\right|\right) \leq \epsilon
$$

\section{Proposition 6.2.}

- The sequence $\left(u_{n}, v_{n}, w_{n}\right)$ built in algorithm 1 converges to some $(\hat{u}, \hat{v}, \hat{w})$ minimizer of (6.1).

- The sequence $\left(u_{n}, v_{n}, w_{n}\right)$ built in algorithm 2 is such that $u_{n}$ converges to $\hat{u} \in X$, and if $(\hat{v}, \hat{w})$ is a cluster point of $\left(v_{n}, w_{n}\right)$ then $(\hat{u}, \hat{v}, \hat{w})$ is a minimizer of (6.1).

Proof We first remark that, as we solve successive minimization problems, we have:

$$
F\left(u_{n}, v_{n}, w_{n}\right) \geq F\left(u_{n}, v_{n}, w_{n+1}\right) \geq F\left(u_{n}, v_{n+1}, w_{n+1}\right) \geq F\left(u_{n+1}, v_{n+1}, w_{n+1}\right)
$$

In particular, the sequence $F\left(u_{n}, v_{n}, w_{n}\right)$ is nonincreasing. As it is bounded from below by 0 , it thus converges in $\mathbb{R}$. We denote by $m$ its limit. We want to show that

$$
m=\inf _{(u, v, w) \in X \times X \times X} F(u, v, w)
$$


Without any restriction, we can assume that, $\forall n,\left(u_{n}, v_{n}, w_{n}\right) \in X \times \mu B_{G} \times$ $\delta B_{E}$. As $F$ is coercive and as the sequence $F\left(u_{n}, v_{n}, w_{n}\right)$ converges, we deduce that the sequence $\left(u_{n}, v_{n}, w_{n}\right)$ is bounded in $X \times \mu B_{G} \times \delta B_{E}$. We can thus extract a subsequence $\left(u_{n_{k}}, v_{n_{k}}, w_{n_{k}}\right)$ which converges to $(\hat{u}, \hat{v}, \hat{w})$ as $n_{k} \rightarrow+\infty$, with $(\hat{u}, \hat{v}, \hat{w}) \in X \times \mu B_{G} \times \delta B_{E}$. Moreover, we have, for all $n_{k} \in \mathbb{N}$ and all $w$ in $X$ :

$$
F\left(u_{n_{k}}, v_{n_{k}}, w_{n_{k}+1}\right) \leq F\left(u_{n_{k}}, v_{n_{k}}, w\right)
$$

We also have, for all $n_{k} \in \mathbb{N}$ and all $v$ in $X$ :

$$
F\left(u_{n_{k}}, v_{n_{k}+1}, w_{n_{k}+1}\right) \leq F\left(u_{n_{k}}, v, w_{n_{k}+1}\right)
$$

and for all $n_{k} \in \mathbb{N}$ and all $u$ in $X$ :

$$
F\left(u_{n_{k}}, v_{n_{k}}, w_{n_{k}}\right) \leq F\left(u, v_{n_{k}}, w_{n_{k}}\right)
$$

Let us denote by $\tilde{w}$ a cluster point of $\left(w_{n_{k}+1}\right)$. Considering (6.24), we get (since $F$ is continuous on $X \times \mu B_{G} \times \delta B_{E}$ ):

$$
m=F(\hat{u}, \hat{v}, \hat{w})=F(\hat{u}, \hat{v}, \tilde{w})
$$

By passing to the limit in (6.12), we get: $\tilde{w}=P_{\delta B_{E}}(f-\hat{u}-\hat{v})$. But from (6.29), we know that: $\|f-\hat{u}-\hat{v}-\hat{w}\|=\|f-\hat{u}-\hat{v}-\tilde{w}\|$. By uniqueness of the projection, we conclude that $\tilde{w}=\hat{w}$. Hence $w_{n_{k}+1} \rightarrow \hat{w}$.

Let us denote by $\tilde{v}$ a cluster point of $\left(v_{n_{k}+1}\right)$. In the same way as above, we can show that $\tilde{v}=\hat{v}$. Hence $v_{n_{k}+1} \rightarrow \hat{v}$.

By passing to the limit in (6.26) ( $F$ is continuous on $X \times \mu B_{G} \times \delta B_{E}$ ), we therefore have for all $w$ :

$$
F(\hat{u}, \hat{v}, \hat{w}) \leq F(\hat{u}, \hat{v}, w)
$$

By passing to the limit in (6.27) ( $F$ is continuous on $X \times \mu B_{G} \times \delta B_{E}$ ), we therefore have for all $v$ :

$$
F(\hat{u}, \hat{v}, \hat{w}) \leq F(\hat{u}, v, \hat{w})
$$

And by passing to the limit in (6.28), for all $u$ :

$$
F(\hat{u}, \hat{v}, \hat{w}) \leq F(u, \hat{v}, \hat{w})
$$

$\mathrm{RR} \mathrm{n}^{\circ} 5130$ 
(6.30), (6.31) and (6.32) can respectively be rewritten:

$$
\begin{aligned}
& F(\hat{u}, \hat{v}, \hat{w})=\inf _{w \in X} F(\hat{u}, \hat{v}, w) \\
& F(\hat{u}, \hat{v}, \hat{w})=\inf _{v \in X} F(\hat{u}, v, \hat{w}) \\
& F(\hat{u}, \hat{v}, \hat{w})=\inf _{u \in X} F(u, \hat{v}, \hat{w})
\end{aligned}
$$

But, from the definition of $F(u, v, w),(6.33)$ is equivalent to (see [10]):

$$
0 \in-f+\hat{u}+\hat{v}+\hat{w}+\lambda \partial B^{*}\left(\frac{\hat{w}}{\delta}\right)
$$

and (6.34) to

$$
0 \in-f+\hat{u}+\hat{v}+\hat{w}+\lambda \partial J^{*}\left(\frac{\hat{v}}{\mu}\right)
$$

and (6.35) to

$$
0 \in-f+\hat{u}+\hat{v}+\hat{w}+\lambda \partial J(\hat{u})
$$

The subdifferential of $F$ at $(\hat{u}, \hat{v}, \hat{w})$ is given by:

$$
\partial F(\hat{u}, \hat{v})=\frac{1}{\lambda}\left(\begin{array}{l}
-f+\hat{u}+\hat{v}+\hat{w}+\lambda \partial J(\hat{u}) \\
-f+\hat{u}+\hat{v}+\hat{w}+\lambda \partial J^{*}\left(\frac{\hat{v}}{\mu}\right) \\
-f+\hat{u}+\hat{v}+\hat{w}+\lambda \partial B^{*}\left(\frac{\hat{w}}{\delta}\right)
\end{array}\right)
$$

And thus, according to (6.38), (6.37) and (6.36), we have:

$$
\left(\begin{array}{l}
0 \\
0 \\
0
\end{array}\right) \in \partial F(\hat{u}, \hat{v}, \hat{w})
$$

which is equivalent to: $F(\hat{u}, \hat{v}, \hat{w})=\inf _{(u, v, w) \in X^{3}} F(u, v, w)=m$. Hence the whole sequence $F\left(u_{n}, v_{n}, w_{n}\right)$ converges towards $m$ the minimum of $F$ on $X \times$ $\mu B_{G} \times \delta B_{E}$. From Proposition 6.1 , we deduce that the sequence $\left(u_{n}\right)$ converges to $\hat{u}$ and that $\left(v_{n}+w_{n}\right)$ converges to $\hat{v}+\hat{w}$ when $n$ tends to $+\infty$.

We now turn our attention to the second part of the algorithm. We have:

$$
w_{n+1}=P_{\delta B_{E}}\left(f-\hat{u}-v_{n}\right)
$$




$$
v_{n+1}=P_{\mu B_{G}}\left(f-\hat{u}-w_{n+1}\right)
$$

This transformation is 1-Lipschitz, that is $w_{n+1}=\psi_{1}\left(w_{n}\right)$ and $v_{n+1}=$ $\psi_{1}\left(v_{n}\right)$ with $\psi_{1}$ and $\psi_{2}$ 1-Lipschitz functions: $\psi_{1}(w)=P_{\delta B_{E}}\left(f-\hat{u}-P_{\mu B_{G}}(f-\right.$ $\hat{u}-w))$ and $\psi_{2}(v)=P_{\mu B_{G}}\left(f-\hat{u}-P_{\delta B_{E}}(f-\hat{u}-v)\right)$. With the same arguments as in the first part of the proof, one can extract a subsequence $\left(v_{n_{k}}, w_{n_{k}}\right)$ from $\left(v_{n}, w_{n}\right)$ converging to some $(\hat{v}, \hat{w}) \in \mu B_{G} \times \delta B_{E}$. Moreover, $\psi_{1}(\hat{w})=\hat{w}$ and $\psi_{2}(\hat{v})=\hat{v}$. From this, we deduce that the whole sequence $\left(v_{n}, w_{n}\right)$ converges to $(\hat{v}, \hat{w})$.

\subsection{Limit problem}

Let us introduce the two following problems:

$$
\begin{gathered}
\inf _{f=u+v+w} J(u)+J^{*}\left(\frac{v}{\mu}\right)+B^{*}\left(\frac{w}{\delta}\right) \\
\inf _{f=u+v+w} J(u)+\alpha\|v\|_{G}+\beta\|w\|_{E}
\end{gathered}
$$

We first make the link between (6.44) and (6.43).

Proposition 6.3. Let us fix $\alpha$ and $\beta$ in problem (6.44). Let $(\hat{u}, \hat{v}, \hat{w})$ be a solution of problem (6.44). Choose $\mu=\|v\|_{G}$ and $\delta=\|w\|_{E}$ in (6.43). Then $(\hat{u}, \hat{v}, \hat{w})$ is also a solution of problem (6.43).

Now we make the link between (6.43) and (6.1), by checking what happens when $\lambda$ goes to 0 in problem (6.1).

Proposition 6.4. Let us denote by $\left(u_{\lambda_{n}}, v_{\lambda_{n}}, w_{\lambda_{n}}\right)$ a solution of problem (6.1) (with $\lambda=\lambda_{n}$ ). Then, when $\lambda_{n}$ goes to 0 , any cluster point of the sequence $\left(u_{\lambda_{n}}, v_{\lambda_{n}}, w_{\lambda_{n}}\right)$ is a solution of problem (6.43).

The proofs are straightforward generalizations of the proofs in [4].

\subsection{Numerical results}

We display here some numerical results.

$\mathrm{RR} \mathrm{n}^{\circ} 5130$ 
Choice of the parameters: The parameter $\lambda$ controls the $L^{2}$ norm of the residual $f-u-v$. The smaller it is, the smaller the norm of the residual becomes. In practice, we just need to set $\lambda$ small (in our experiments, we have used $\lambda \leq 1.0$ : the $L^{\infty}$ norm of the residual is then smaller than 1.5 (and the gray values of our images go from 0 to 255)).

The parameter $\mu$ represents the $G$ norm of the $v$ component.

The parameter $\delta$ represents the $E$ norm of the $w$ component. Having a closer look at the algorithm (as in Subsection5), we see that $2 \delta$ is the threshold which is used in the waveletsoft-thresholding. In the case of denoising, we can then use the classical threshold $\sigma \sqrt{2 \log \left(N^{2}\right)}$ where $N^{2}$ is the size of the image $[9,13,16]$. We therefore choose $\delta=\frac{\sigma \sqrt{2 \log \left(N^{2}\right)}}{2}$. In fact, to get better results, we have introduced a weighting parameter $\eta$, and we set $\delta=\eta \frac{\sigma \sqrt{2 \log \left(N^{2}\right)}}{2}$. In practice, $\eta$ is equal to 1 or slightly smaller.

We display some numerical results on Figures 12 to 16 .

Comments The model in [19] can be written:

$$
\min _{u, v}\left(\|\mathcal{C} u\|_{1}+\|\mathcal{D} v\|_{1}+\alpha\|f-u-v\|_{2}^{2}+\beta J(u)\right)
$$

where $\mathcal{C}$ is a transform adapted to the geometrical part of the image (for instance the curvelet transform), and $\mathcal{D}$ a transform adapted to the textured part of the image (for instance the discrete cosinus transform). The noise $w=f-u-v$ is penalized through its $L^{2}$ norm. In fact, the authors of [19] get the noise component as a by-product of their model. But they have not really introduce a norm specific to the noise, contrary to what we have done. Moreover, if the curvelet transform is being used for the geometrical part of the image in (6.45), the computation time is then much longer than with our algorithm.

Acknowledgement: Most of this work was done while the second author was still with CEREMADE, CNRS UMR 7534, Université de Paris Dauphine, Paris, France. The first author thanks the hospitality of CEREMADE where this work was initiated. Part of this work has been funded by GdR-PrC ISIS (http://www-isis.enst.fr/) through the young researcher program. 


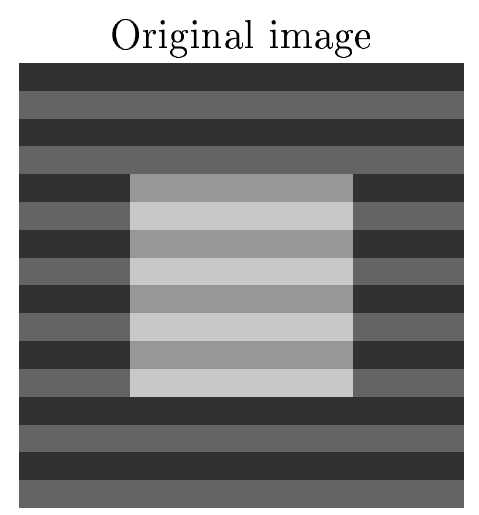

$u$

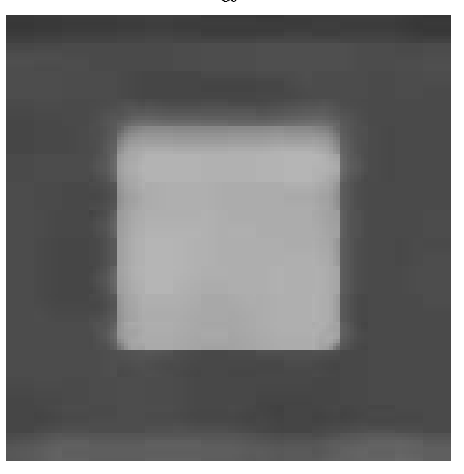

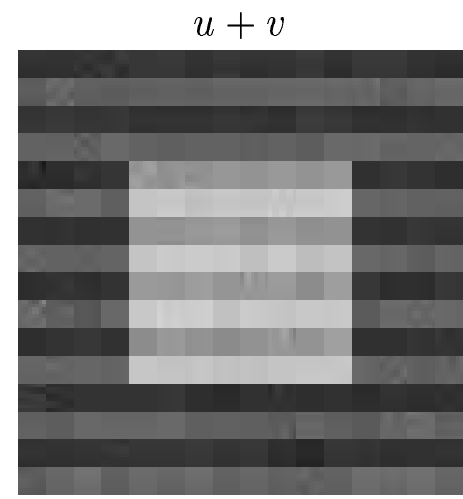

$w+150$
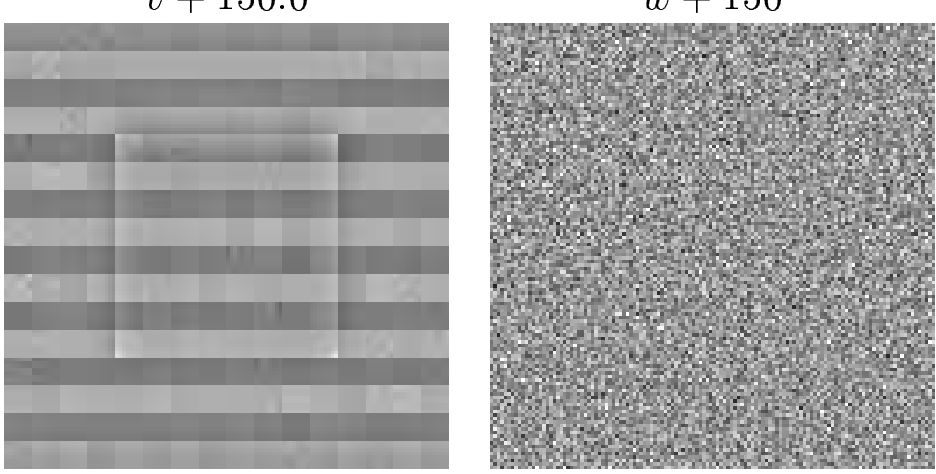

Figure 12: A simple example $(\lambda=0.5, \mu=120, \eta=1.0$, Haar $)$ 

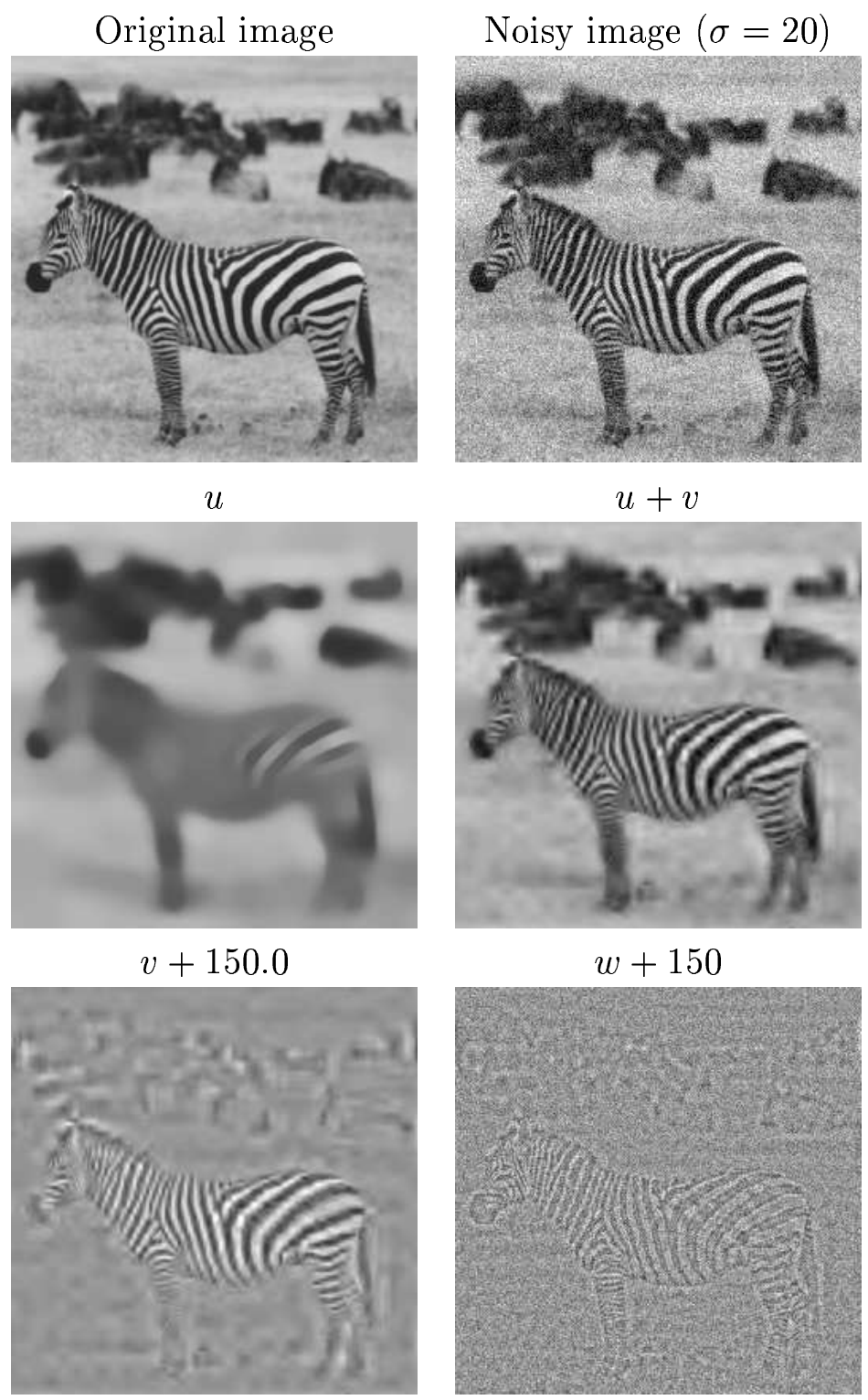

Figure 13: Zebra image $(\lambda=1.0, \mu=100, \eta=0.7$, Daub8) 

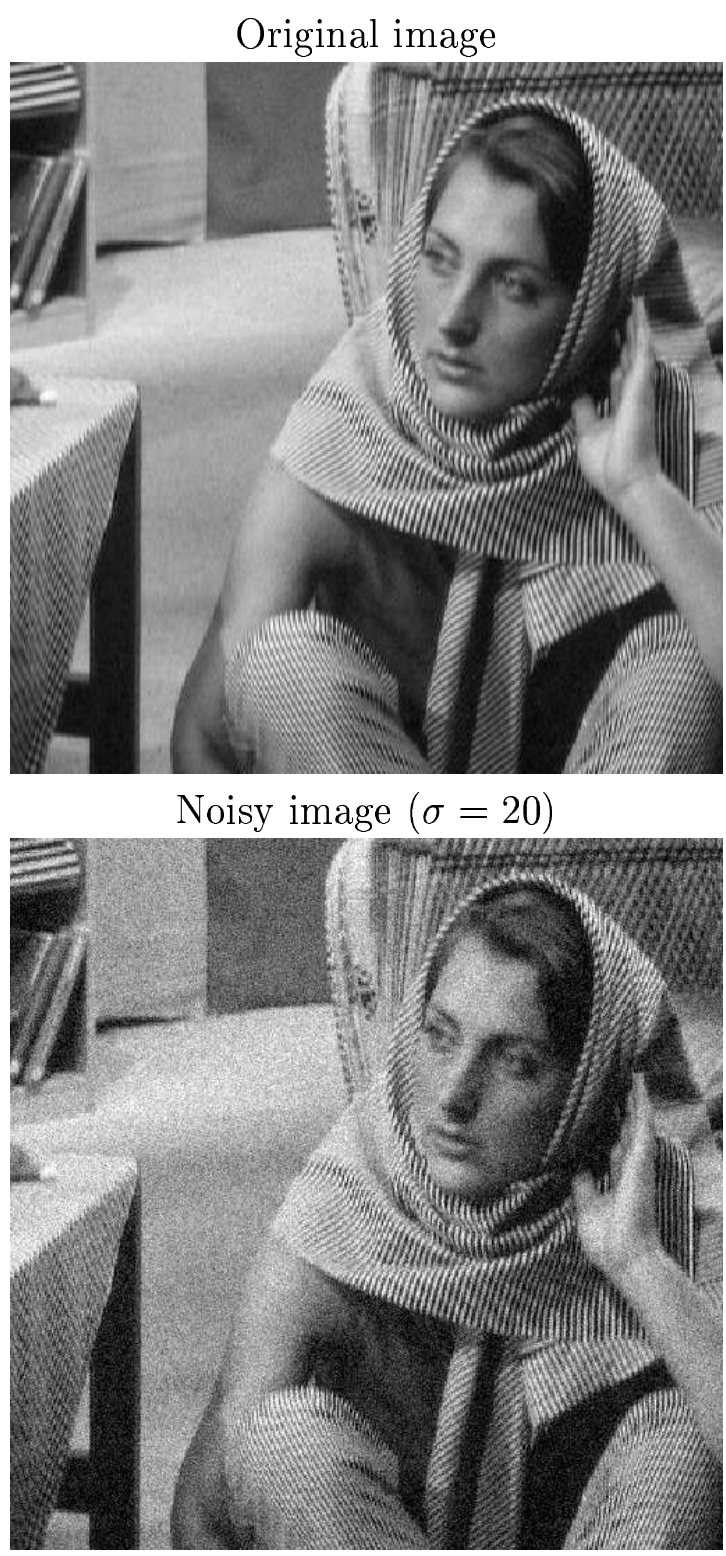

Figure 14: Barbara image I

$\mathrm{RR} \mathrm{n}^{\circ} 5130$ 

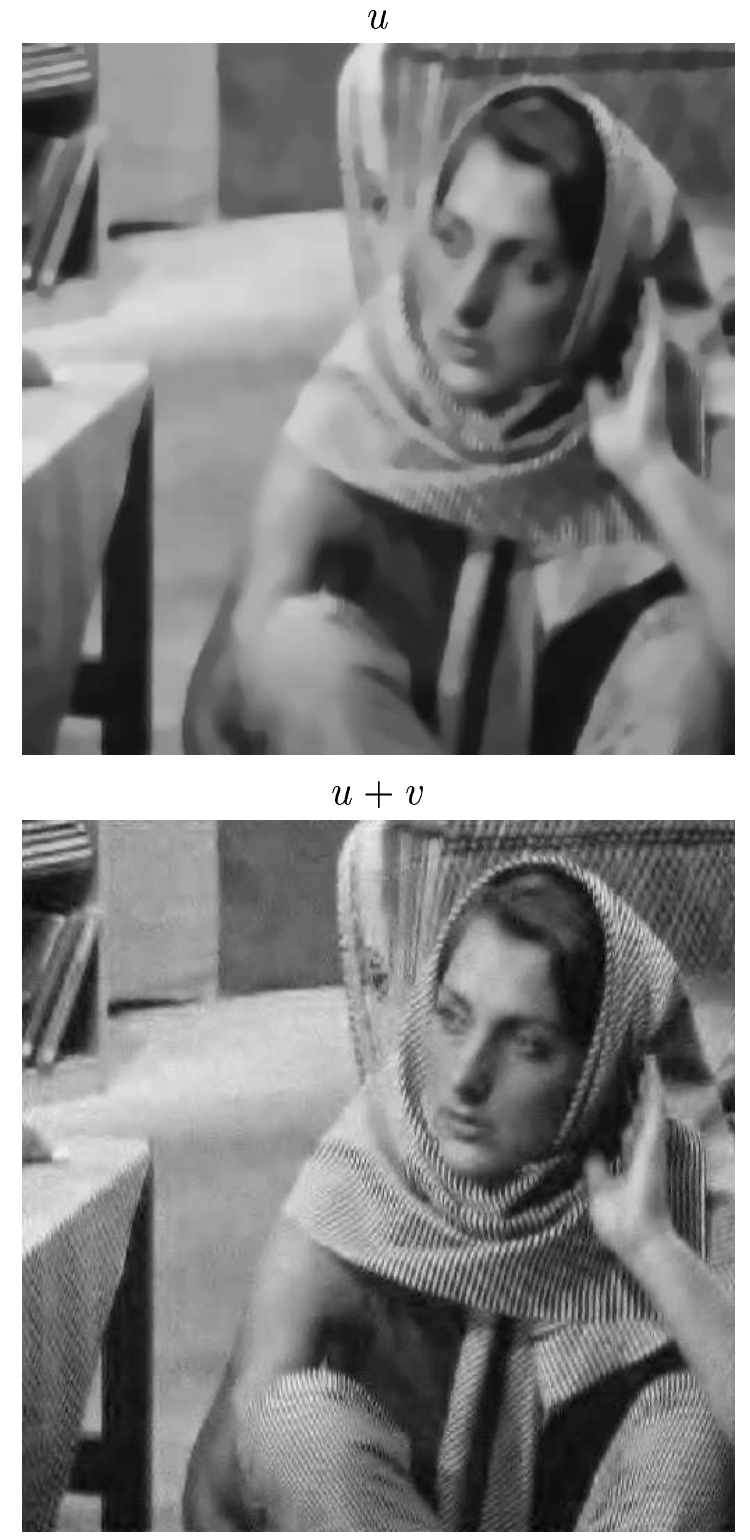

Figure 15: Barbara image II $(\lambda=1.0, \mu=30, \eta=0.6$, Daub8) 


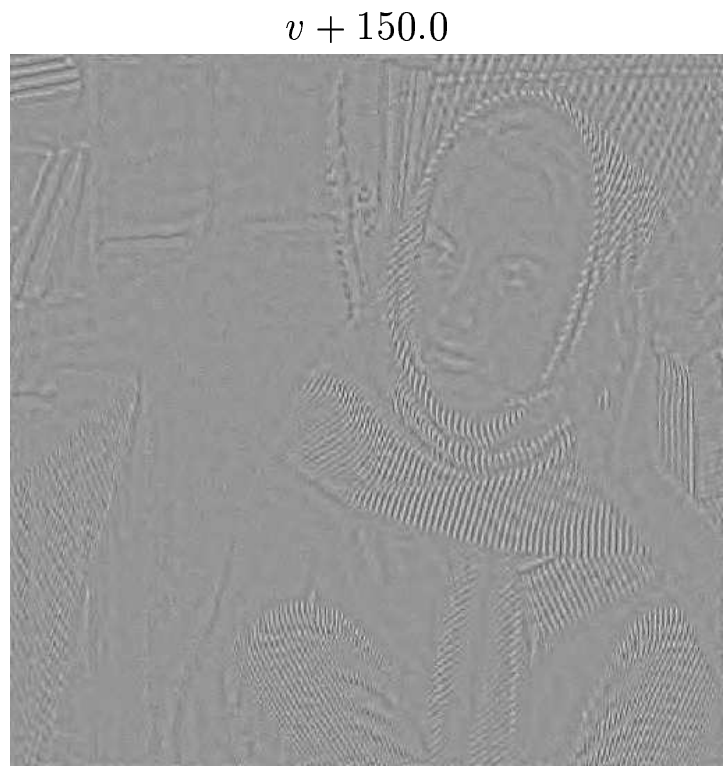

$$
w+150
$$

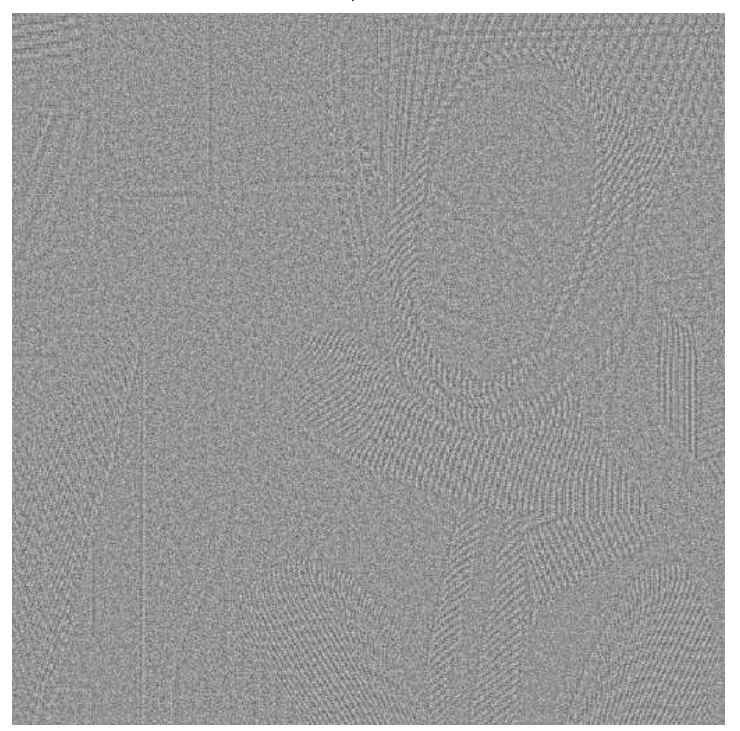

Figure 16: Barbara image III $(\lambda=1.0, \mu=30, \eta=0.6$, Daub8)

RR $n^{\circ} 5130$ 


\section{References}

[1] R. Adams. Sobolev Spaces. Pure and applied Mathematics. Academic Press, Inc, 1975.

[2] L. Ambrosio, N. Fusco, and D. Pallara. Functions of bounded variations and free discontinuity problems. Oxford mathematical monographs. Oxford University Press, 2000.

[3] G. Aubert and P. Kornprobst. Mathematical Problems in Image Processing, volume 147 of Applied Mathematical Sciences. Springer-Verlag, 2002.

[4] J.F. Aujol, G. Aubert, L. Blanc-Féraud, and A. Chambolle. Decomposing an image: Application to textured images and SAR images, 2003. INRIA Research Report 4704, to appear in JMIV, http://www.inria.fr/rrrt/rr4704.html.

[5] J.F. Aujol, G. Aubert, L. Blanc-Féraud, and A. Chambolle. Decomposing an image: Application to SAR images. In Scale-Space '03, volume 1682 of Lecture Notes in Computer Science, 2003.

[6] A. Chambolle. An algorithm for total variation minimization and applications. JMIV, 20:89-97, 2004.

[7] A. Chambolle and P.L. Lions. Image recovery via total variation minimization and related problems. Numerische Mathematik, 76(3):167-188, 1997.

[8] A. Chambolle, R.A. De Vore, N. Lee, and B.J. Lucier. Nonlinear wavelet image processing: Variational problems, compression, and noise removal through wavelet shrinkage. IEEE Transcations on Image Processing, 7(3):319-335, March 1998.

[9] D.L. Donoho and M. Johnstone. Adapting to unknown smoothness via wavelet shrinkage. Journal of the American Statistical Association, 90(432):1200-1224, December 1995.

[10] I. Ekeland and R. Temam. Analyse convexe et problèmes variationnels. Etudes Mathématiques. Dunod, 1974. 
[11] L.C. Evans. Partial Differential Equations, volume 19 of Graduate Studies in Mathematics. American Mathematical Society, 1991.

[12] F. Malgouyres. Mathematical analysis of a model which combines total variation and wavelet for image restoration. Journal of information processes, 2(1):1-10, 2002.

[13] F. Malgouyres. Minimizing the total variation under a general convex constraint for image restoration. IEEE transactions on image processing, 11(12):1450-1456, December 2002.

[14] S.G. Mallat. A theory for multiresolutionsignal decomposition: The wavelet representation. IEEE Transactions on Pattern Analysis and Machine Intelligence, 11(7):674-693, July 1989.

[15] S.G. Mallat. A Wavelet Tour of Signal Processing. Academic Press, 1998.

[16] Yves Meyer. Oscillating patterns in image processing and in some nonlinear evolution equations, March 2001. The Fifteenth Dean Jacquelines B. Lewis Memorial Lectures.

[17] S.J. Osher, A. Sole, and L.A. Vese. Image decomposition and restoration using total variation minimization and the $\mathrm{H}^{-1}$ norm. Multiscale Modeling and Simulation: A SIAM Interdisciplinary Journal, 1(3):349-370, 2003.

[18] L. Rudin, S. Osher, and E. Fatemi. Nonlinear total variation based noise removal algorithms. Physica D, 60:259-268, 1992.

[19] J.L. Starck, M. ELad, and D.L. Donoho. Image decomposition: separation of texture from piecewise smooth content, 2003. To appear in IEEE Transactions on Image Processing.

[20] L.A. Vese and S.J. Osher. Modeling textures with total variation minimization and oscillating patterns in image processing. Journal of Scientific Computing, 19:553-572, 2003.

$\mathrm{RR} \mathrm{n}^{\circ} 5130$ 


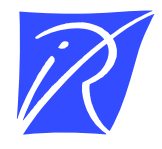

\section{Unité de recherche INRIA Sophia Antipolis 2004, route des Lucioles - BP 93 - 06902 Sophia Antipolis Cedex (France)}

Unité de recherche INRIA Futurs : Parc Club Orsay Université - ZAC des Vignes 4, rue Jacques Monod - 91893 ORSAY Cedex (France)

Unité de recherche INRIA Lorraine : LORIA, Technopôle de Nancy-Brabois - Campus scientifique 615, rue du Jardin Botanique - BP 101 - 54602 Villers-lès-Nancy Cedex (France)

Unité de recherche INRIA Rennes : IRISA, Campus universitaire de Beaulieu - 35042 Rennes Cedex (France)

Unité de recherche INRIA Rhône-Alpes : 655, avenue de l'Europe - 38334 Montbonnot Saint-Ismier (France)

Unité de recherche INRIA Rocquencourt : Domaine de Voluceau - Rocquencourt - BP 105 - 78153 Le Chesnay Cedex (France)

INRIA - Domaine de Voluceau - Rocquencourt, BP 105 - 78153 Le Chesnay Cedex (France)

http://www.inria.fr

ISSN 0249-6399 